\title{
A Study of Charm Meson Production in Semileptonic B Decays
}

\section{The OPAL Collaboration}

\begin{abstract}
The results of a study of charm meson production in semileptonic B hadron decays are presented. Based on a sample of 1.72 million hadronic $\mathrm{Z}^{0}$ decays the following product branching ratios (averaged over electrons and muons) are obtained :

$$
\begin{aligned}
B\left(b \rightarrow \mathrm{D}^{+} \ell X\right) \cdot B\left(\mathrm{D}^{+} \rightarrow \mathrm{K}^{-} \pi^{+} \pi^{+}\right) & =(1.82 \pm 0.20 \pm 0.12) \times 10^{-3} \\
B\left(b \rightarrow \mathrm{D}^{0} \ell X\right) \cdot B\left(\mathrm{D}^{0} \rightarrow \mathrm{K}^{-} \pi^{+}\right) & =(2.52 \pm 0.14 \pm 0.17) \times 10^{-3} \\
B\left(b \rightarrow \mathrm{D}^{*+} \ell X\right) \cdot B\left(\mathrm{D}^{*+} \rightarrow \mathrm{D}^{0} \pi^{+}\right) \cdot B\left(\mathrm{D}^{0} \rightarrow \mathrm{K}^{-} \pi^{+}\right) & =(7.53 \pm 0.47 \pm 0.56) \times 10^{-4}
\end{aligned}
$$

where the first error is statistical and the second systematic.

The results of a search for the narrow $\mathrm{P}$-wave charmed mesons, $\left(\mathrm{D}_{J}\right)$, in semileptonic $\mathrm{B}$ decays are also reported. Using the decay channels $\mathrm{D}_{J}^{0} \rightarrow \mathrm{D}^{+} \pi^{-}, \mathrm{D}_{J}^{0} \rightarrow$ $\mathrm{D}^{*+} \pi^{-}$and $\mathrm{D}_{J}^{+} \rightarrow \mathrm{D}^{0} \pi^{+}$we observe a total signal of $44 \pm 8_{-7}^{+3} \mathrm{D}_{J}^{0}$ and $48 \pm 10_{-6}^{+3}$ $\mathrm{D}_{J}^{+}$events. This is the first evidence for semileptonic $\mathrm{B}$ decays to charged $\mathrm{P}$-wave charm states. The observed signals also provide evidence for the production of both pseudovector and tensor $\mathrm{P}$-wave charmed mesons in semileptonic B decay. Together these modes are estimated to make up $34 \pm 7 \%$ of charmed semileptonic $\mathrm{B}^{0}$ and $\mathrm{B}^{+}$ decays.
\end{abstract}

\section{Submitted to Zeit für Physik}


R. Akers ${ }^{16}$, G. Alexander ${ }^{23}$, J. Allison ${ }^{16}$, K. Ametewee ${ }^{25}$, K.J.Anderson ${ }^{9}$, S. Arcelli ${ }^{2}$, S. Asai ${ }^{24}$, D. Axen ${ }^{29}$, G. Azuelos ${ }^{18, a}$, A.H. Ball ${ }^{17}$, E. Barberio ${ }^{26}$, R.J. Barlow ${ }^{16}$, R. Bartoldus ${ }^{3}$, J.R. Batley ${ }^{5}$, G. Beaudoin ${ }^{18}$, A. Beck ${ }^{23}$, G.A.Beck ${ }^{13}$, C. Beeston ${ }^{16}$, T.Behnke ${ }^{27}$, K.W.Bell ${ }^{20}$, G. Bella ${ }^{23}$, S. Bentvelsen ${ }^{8}$, P. Berlich ${ }^{10}$, S. Bethke ${ }^{32}$, O. Biebel ${ }^{32}$, I.J. Bloodworth ${ }^{1}$, P. Bock ${ }^{11}$, H.M. Bosch ${ }^{11}$, M. Boutemeur ${ }^{18}$, S. Braibant ${ }^{12}$, P. Bright-Thomas ${ }^{25}$, R.M. Brown ${ }^{20}$, A. Buijs ${ }^{8}$, H.J. Burckhart ${ }^{8}$, R.Bürgin ${ }^{10}$, C.Burgard ${ }^{27}$, N.Capdevielle ${ }^{18}$, P.Capiluppi ${ }^{2}$, R.K. Carnegie ${ }^{6}$, A.A.Carter ${ }^{13}$, J.R.Carter ${ }^{5}$, C.Y.Chang ${ }^{17}$, C. Charlesworth ${ }^{6}$, D.G.Charlton ${ }^{1, b}$, S.L.Chu ${ }^{4}$, P.E.L. Clarke ${ }^{15}$, J.C.Clayton ${ }^{1}$, S.G.Clowes ${ }^{16}$, I.Cohen ${ }^{23}$, J.E.Conboy ${ }^{15}$, O.C.Cooke ${ }^{16}$, M.Cuffiani ${ }^{2}$, S. Dado ${ }^{22}$, C. Dallapiccola ${ }^{17}$, G.M.Dallavalle ${ }^{2}$, C.Darling ${ }^{31}$, S. De Jong ${ }^{12}$, L.A.del Pozo ${ }^{8}$, H. Deng ${ }^{17}$, M.Dittmar ${ }^{4}$, M.S. Dixit ${ }^{7}$, E. do Couto e Silva ${ }^{12}$, J.E. Duboscq ${ }^{8}$, E. Duchovni ${ }^{26}$, G. Duckeck $^{8}$, I.P. Duerdoth ${ }^{16}$, U.C.Dunwoody ${ }^{5}$, J.E.G. Edwards ${ }^{16}$, P.A. Elcombe ${ }^{5}$, P.G. Estabrooks ${ }^{6}$, E. Etzion ${ }^{23}$, H.G. Evans ${ }^{9}$, F.Fabbri ${ }^{2}$, B.Fabbro ${ }^{21}$, M.Fanti ${ }^{2}$, P.Fath ${ }^{11}$, M.Fierro ${ }^{2}$, M. Fincke-Keeler ${ }^{28}$, H.M. Fischer ${ }^{3}$, P. Fischer ${ }^{3}$, R. Folman ${ }^{26}$, D.G. Fong ${ }^{17}$, M. Foucher ${ }^{17}$, H. Fukui ${ }^{24}$, A.Fürtjes ${ }^{8}$, P. Gagnon ${ }^{6}$, A. Gaidot ${ }^{21}$, J.W.Gary ${ }^{4}$, J. Gascon ${ }^{18}$, N.I. Geddes ${ }^{20}$, C. Geich-Gimbel ${ }^{3}$, S.W.Gensler ${ }^{9}$, F.X.Gentit ${ }^{21}$, T.Geralis ${ }^{20}$, G. Giacomelli ${ }^{2}$, P. Giacomelli ${ }^{4}$, R. Giacomelli ${ }^{2}$, V.Gibson ${ }^{5}$, W.R. Gibson ${ }^{13}$, J.D. Gillies ${ }^{20}$, J. Goldberg ${ }^{22}$, D.M. Gingrich ${ }^{30, a}$, M.J.Goodrick ${ }^{5}$, W.Gorn ${ }^{4}$, C.Grandi ${ }^{2}$, E. Gross ${ }^{26}$, J. Hagemann ${ }^{27}$, G.G.Hanson ${ }^{12}$, M. Hansroul ${ }^{8}$, C.K.Hargrove ${ }^{7}$, P.A.Hart ${ }^{9}$, M.Hauschild ${ }^{8}$, C.M.Hawkes ${ }^{8}$, E. Heflin ${ }^{4}$, R.J. Hemingway ${ }^{6}$, G. Herten ${ }^{10}$, R.D. Heuer ${ }^{8}$, J.C.Hill ${ }^{5}$, S.J.Hillier ${ }^{8}$, T. Hilse ${ }^{10}$, P.R. Hobson ${ }^{25}$, D. Hochman ${ }^{26}$, R.J.Homer ${ }^{1}$, A.K. Honma ${ }^{28, a}$, R. Howard ${ }^{29}$, R.E. Hughes-Jones ${ }^{16}$, P. Igo-Kemenes ${ }^{11}$, D.C. Imrie ${ }^{25}$, A. Jawahery ${ }^{17}$, P.W.Jeffreys ${ }^{20}$, H. Jeremie ${ }^{18}$, M. Jimack ${ }^{1}$, M. Jones ${ }^{6}$, R.W.L.Jones ${ }^{8}$, P. Jovanovic ${ }^{1}$, C. Juii ${ }^{4}$, D. Karlen ${ }^{6}$, J. Kanzaki ${ }^{24}$, K. Kawagoe ${ }^{24}$, T. Kawamoto ${ }^{24}$, R.K. Keeler ${ }^{28}$, R.G. Kellogg ${ }^{17}$, B.W. Kennedy ${ }^{20}$, B. King ${ }^{8}$, J. King ${ }^{13}$, J. Kirk ${ }^{29}$, S. Kluth ${ }^{5}$, T. Kobayashi ${ }^{24}$, M. Kobe ${ }^{10}$, D.S. Koetke ${ }^{6}$, T.P.Kokott ${ }^{3}$, S. Komamiya ${ }^{24}$, R. Kowalewski ${ }^{8}$, T. Kress ${ }^{11}$, P. Krieger ${ }^{6}$, J. von Krogh ${ }^{11}$, P. Kyberd ${ }^{13}$, G.D. Lafferty ${ }^{16}$, H. Lafoux ${ }^{8}$, R. Lahmann ${ }^{17}$, W.P. Lai ${ }^{19}$, J. Lauber ${ }^{8}$, J.G. Layter ${ }^{4}$, P. Leblanc ${ }^{18}$, P. Le Du ${ }^{21}$, A.M. Lee ${ }^{31}$, E. Lefebvre ${ }^{18}$, D. Lellouch ${ }^{26}$, C. Leroy ${ }^{18}$, J. Letts ${ }^{2}$, L. Levinson ${ }^{26}$, Z. Li $^{12}$, S.L. Lloyd ${ }^{13}$, F.K. Loebinger ${ }^{16}$, G.D. Long ${ }^{17}$, B. $\operatorname{Lorazo}^{18}$, M.J. Losty ${ }^{7}$, X.C. Lou ${ }^{8}$, J. Ludwig ${ }^{10}$, A. Luig ${ }^{10}$, M. Mannelli ${ }^{8}$, S. Marcellini ${ }^{2}$, C.Markus ${ }^{3}$, A.J.Martin ${ }^{13}$, J.P.Martin ${ }^{18}$, T. Mashimo ${ }^{24}$, W.Matthews ${ }^{25}$, P.Mättig ${ }^{3}$, U.Maur ${ }^{3}$, J. McKenna ${ }^{29}$, T.J.McMahon ${ }^{1}$, A.I. McNab ${ }^{13}$,

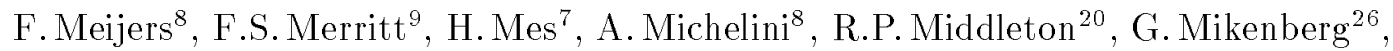
D.J. Miller ${ }^{15}$, R. Mirr $^{26}$, W.Mohr ${ }^{10}$, A.Montanari ${ }^{2}$, T. Mori ${ }^{24}$, M. Moriï ${ }^{24}$, U.Müller ${ }^{3}$, B. Nellen ${ }^{3}$, B. Nijjhar ${ }^{16}$, S.W.O'Neale ${ }^{1}$, F.G.Oakham ${ }^{7}$, F.Odorici ${ }^{2}$, H.O.Ogren ${ }^{12}$, N.J.Oldershaw ${ }^{16}$, C.J.Oram ${ }^{28, a}$, M.J.Oreglia ${ }^{9}$, S. Orito ${ }^{24}$, J.P.Pansart ${ }^{21}$, G.N.Patrick ${ }^{20}$, M.J. Pearce ${ }^{1}$, P.D. Phillips ${ }^{16}$, J.E.Pilcher ${ }^{9}$, J.Pinfold ${ }^{30}$, D.E.Plane ${ }^{8}$, P. Poffenberger ${ }^{28}$, B. Poli ${ }^{2}$, A. Posthaus ${ }^{3}$, T.W.Pritchard ${ }^{13}$, H. Przysiezniak ${ }^{30}$, M.W. Redmond ${ }^{8}$, D.L. Rees ${ }^{8}$, D. Rigby ${ }^{1}$, M.G. Rison ${ }^{5}$, S.A. Robins ${ }^{13}$, D. Robinson ${ }^{5}$, N. Rodning ${ }^{30}$, J.M. Roney ${ }^{28}$, E. Ros ${ }^{8}$, A.M. Rossi $^{2}$, M. Rosvick ${ }^{28}$, P. Routenburg ${ }^{30}$, Y. Rozen ${ }^{8}$, K.Runge ${ }^{10}$, O.Runolfsson ${ }^{8}$, D.R.Rust ${ }^{12}$, M.Sasaki ${ }^{24}$, C.Sbarra ${ }^{2}$, A.D.Schaile ${ }^{8}$, O.Schaile ${ }^{10}$, F.Scharf ${ }^{3}$, P.Scharff-Hansen ${ }^{8}$, P.Schenk ${ }^{4}$, B.Schmitt ${ }^{3}$, M.Schröder ${ }^{8}$, H.C.Schultz-Coulon ${ }^{10}$, P.Schütz ${ }^{3}$, M.Schulz ${ }^{8}$, C.Schwick ${ }^{27}$, J.Schwiening ${ }^{3}$,

W.G.Scott ${ }^{20}$, M.Settles ${ }^{12}$, T.G.Shears ${ }^{5}$, B.C.Shen ${ }^{4}$, C.H.Shepherd-Themistocleous ${ }^{7}$, P.Sherwood ${ }^{15}$, G.P.Siroli ${ }^{2}$, A.Skillman ${ }^{15}$, A.Skuja ${ }^{17}$, A.M.Smith ${ }^{8}$, T.J.Smith ${ }^{28}$, G.A.Snow ${ }^{17}$, R.Sobie ${ }^{28}$, S.Söldner-Rembold ${ }^{10}$, R.W.Springer ${ }^{30}$, M.Sproston ${ }^{20}$, A.Stahl ${ }^{3}$, M.Starks ${ }^{12}$, C.Stegmann ${ }^{10}$, K.Stephens ${ }^{16}$, J.Steuerer ${ }^{28}$, B.Stockhausen ${ }^{3}$, D.Strom ${ }^{19}$, P.Szymanski ${ }^{20}$,

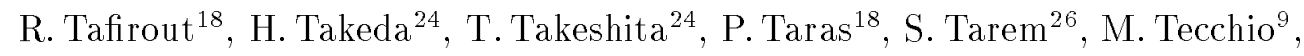


P.Teixeira-Dias ${ }^{11}$, N. Tesch ${ }^{3}$, M.A.Thomson ${ }^{8}$, O. Tousignant $^{18}$, S. Towers $^{6}$, M. Tscheulin $^{10}$, T. Tsukamoto ${ }^{24}$, A. Turcot ${ }^{9}$, M.F. Turner-Watson ${ }^{8}$, P. Utzat ${ }^{11}$, R. Van Kooten ${ }^{12}$, G. Vasseur ${ }^{21}$, P. Vikas ${ }^{18}$, M. Vincter ${ }^{28}$, A. Wagner ${ }^{27}$, D.L. Wagner ${ }^{9}$, C.P. Ward ${ }^{5}$, D.R. Ward $^{5}$, J.J. Ward ${ }^{15}$, P.M. Watkins ${ }^{1}$, A.T. Watson ${ }^{1}$, N.K. Watson ${ }^{7}$, P. Weber ${ }^{6}$, P.S. Wells ${ }^{8}$, N. Wermes ${ }^{3}$, B. Wilkens ${ }^{10}$, G.W.Wilson ${ }^{27}$, J.A.Wilson ${ }^{1}$, V-H.Winterer ${ }^{10}$, T. Wlodek ${ }^{26}$, G. Wolf ${ }^{26}$, S. Wotton ${ }^{11}$, T.R. Wyatt ${ }^{16}$, A. Yeaman ${ }^{13}$, G. Yekutieli ${ }^{26}$, M. Yurko ${ }^{18}$, V.Zacek ${ }^{18}$, W.Zeuner ${ }^{8}$, G.T.Zorn ${ }^{17}$.

${ }^{1}$ School of Physics and Space Research, University of Birmingham, Birmingham B15 2TT, UK ${ }^{2}$ Dipartimento di Fisica dell' Università di Bologna and INFN, I-40126 Bologna, Italy

${ }^{3}$ Physikalisches Institut, Universität Bonn, D-53115 Bonn, Germany

${ }^{4}$ Department of Physics, University of California, Riverside CA 92521, USA

${ }^{5}$ Cavendish Laboratory, Cambridge CB3 0HE, UK

${ }^{6}$ Carleton University, Department of Physics, Colonel By Drive, Ottawa, Ontario K1S 5B6, Canada

${ }^{7}$ Centre for Research in Particle Physics, Carleton University, Ottawa, Ontario K1S 5B6, Canada

${ }^{8} \mathrm{CERN}$, European Organisation for Particle Physics, CH-1211 Geneva 23, Switzerland

${ }^{9}$ Enrico Fermi Institute and Department of Physics, University of Chicago, Chicago IL 60637, USA

${ }^{10}$ Fakultät für Physik, Albert Ludwigs Universität, D-79104 Freiburg, Germany

${ }^{11}$ Physikalisches Institut, Universität Heidelberg, D-69120 Heidelberg, Germany

${ }^{12}$ Indiana University, Department of Physics, Swain Hall West 117, Bloomington IN 47405, USA

${ }^{13}$ Queen Mary and Westfield College, University of London, London E1 4NS, UK

${ }^{15}$ University College London, London WC1E 6BT, UK

${ }^{16}$ Department of Physics, Schuster Laboratory, The University, Manchester M13 9PL, UK

${ }^{17}$ Department of Physics, University of Maryland, College Park, MD 20742, USA

${ }^{18}$ Laboratoire de Physique Nucléaire, Université de Montréal, Montréal, Quebec H3C 3J7, Canada

${ }^{19}$ University of Oregon, Department of Physics, Eugene OR 97403, USA

${ }^{20}$ Rutherford Appleton Laboratory, Chilton, Didcot, Oxfordshire OX11 0QX, UK

${ }^{21} \mathrm{CEA}$, DAPNIA/SPP, CE-Saclay, F-91191 Gif-sur-Yvette, France

${ }^{22}$ Department of Physics, Technion-Israel Institute of Technology, Haifa 32000, Israel

${ }^{23}$ Department of Physics and Astronomy, Tel Aviv University, Tel Aviv 69978, Israel

${ }^{24}$ International Centre for Elementary Particle Physics and Department of Physics, University of Tokyo, Tokyo 113, and Kobe University, Kobe 657, Japan

${ }^{25}$ Brunel University, Uxbridge, Middlesex UB8 3PH, UK

${ }^{26}$ Particle Physics Department, Weizmann Institute of Science, Rehovot 76100, Israel

${ }^{27}$ Universität Hamburg/DESY, II Institut für Experimental Physik, Notkestrasse 85, D-22607 Hamburg, Germany

${ }^{28}$ University of Victoria, Department of Physics, P O Box 3055, Victoria BC V8W 3P6, Canada ${ }^{29}$ University of British Columbia, Department of Physics, Vancouver BC V6T 1Z1, Canada

${ }^{30}$ University of Alberta, Department of Physics, Edmonton AB T6G 2J1, Canada

${ }^{31}$ Duke University, Dept of Physics, Durham, NC 27708-0305, USA

${ }^{32}$ Technische Hochschule Aachen, III Physikalisches Institut, Sommerfeldstrasse 26-28, D-52056 Aachen, Germany

${ }^{a}$ Also at TRIUMF, Vancouver, Canada V6T $2 \mathrm{~A} 3$

${ }^{b}$ a Royal Society University Research Fellow 


\section{Introduction}

It is well established that a large fraction of semileptonic $\mathrm{B}^{0}$ and $\mathrm{B}^{+}$decays to charmed mesons are not accounted for by the measured exclusive decays $\mathrm{B}^{0} \rightarrow \mathrm{D}^{-} \ell^{+} \nu, \mathrm{B}^{0} \rightarrow$ $\mathrm{D}^{*-} \ell^{+} \nu, \mathrm{B}^{+} \rightarrow \overline{\mathrm{D}}^{0} \ell^{+} \nu$ and $\mathrm{B}^{+} \rightarrow \overline{\mathrm{D}}^{\star 0} \ell^{+} \nu[1]$. Given that several theoretical models predict that these exclusive modes should be dominant $[2,3]$ and the difficulties in understanding of the total semileptonic rate [4] it is of interest to search for and measure other exclusive semileptonic decays. The remaining exclusive semileptonic $\mathrm{B}^{0}$ and $\mathrm{B}^{+}$charmed decay modes may consist of decays to $\mathrm{P}$-wave charmed mesons, higher spin states and nonresonant $\mathrm{B} \rightarrow \mathrm{D}^{(*)} n(\pi) \ell^{+} \nu$ decays.

One method of understanding the contribution from these various processes is to measure the relative rates of the different charmed mesons in semileptonic $b$ decays. At LEP these measurements are more complicated than at $\Upsilon(4 \mathrm{~S})$ energies because $\mathrm{B}_{s}$ mesons and $b$ baryons are produced in addition to the $\mathrm{B}^{0}$ and $\mathrm{B}^{+}$mesons. However, as $\mathrm{B}_{s}$ mesons and $b$ baryons are expected to decay only rarely to semileptonic decay modes containing $\mathrm{D}^{0}, \mathrm{D}^{+}$or $\mathrm{D}^{*+}$ mesons, measurements of these rates allow one to determine the fraction of $b$ quarks which fragment into a $\mathrm{B}^{0}$ or $\mathrm{B}^{+}$meson.

In addition it is possible to search directly for the production of excited charmed states in semileptonic $b$ decays. Experimentally the most accessible states are the narrow P-wave mesons $\mathrm{D}_{1}^{0}(2420), \mathrm{D}_{2}^{* 0}(2460), \mathrm{D}_{1}^{+}(2420)^{1}$ and $\mathrm{D}_{2}^{*+}(2460)$. These have natural widths of about $20 \mathrm{MeV}$ and have been observed in $\mathrm{D} \pi$ and $\mathrm{D}^{*} \pi$ decay modes [5-9]. The ARGUS collaboration has published evidence [10] for the production of neutral P-wave mesons in semileptonic B meson decays. These measurements are experimentally difficult because of the large combinatorial backgrounds. At LEP, the boost of the $b$ hadrons combined with precise vertex reconstruction allows a significant reduction in the combinatorial backgrounds and is expected to allow relatively pure samples of these decays to be isolated.

In this paper we present a high statistics measurement of the inclusive rates $b \rightarrow D^{0} \ell \mathrm{X}$, $b \rightarrow \mathrm{D}^{+} \ell \mathrm{X}$ and $b \rightarrow \mathrm{D}^{*+} \ell \mathrm{X}$ in semileptonic $\mathrm{B}$ decays at $\mathrm{Z}^{0}$ energies. We also report on the results of a search for the narrow P-wave charmed mesons in semileptonic $b$ decays.

Throughout this paper, charge conjugation is implicitly assumed and the symbol $\mathrm{D}^{(*)}$ denotes either a $\mathrm{D}^{0} / \mathrm{D}^{+}$or a $\mathrm{D}^{*}(2010)^{0} / \mathrm{D}^{*}(2010)^{+}$meson. The symbol $\mathrm{D}_{J}$ is used to denote a mixture of $\mathrm{P}$-wave charmed mesons.

\section{The OPAL detector}

A complete description of the OPAL detector may be found elsewhere [11-13]. We describe briefly the aspects of the detector pertinent to this analysis. Charged particle tracking is performed by the central detector which consists of a large volume jet chamber, a precision vertex drift chamber and chambers measuring the $z$-coordinate ${ }^{2}$ of tracks as they leave the jet chamber. In 1991 this tracking system was enhanced by the addition of a silicon microvertex detector [12] providing $r-\phi$ coordinate measurements. For the 1993 run this device was replaced by an improved detector providing additional $z$ coordinate measurements, but only the $r-\phi$ information is used in this analysis. The

\footnotetext{
${ }^{1}$ The PDG currently refers to this state as the $\mathrm{D}_{J}^{+}(2440)$.

${ }^{2}$ The OPAL coordinate system is defined with positive $z$ being along the electron beam direction, $\theta$ and $\phi$ being the polar and azimuthal angles respectively.
} 
$r-\phi$ tracking precision provided by both silicon detectors is almost identical with spacepoint resolutions, including alignment uncertainties, of about $9 \mu \mathrm{m}$. When combined with the angle and curvature information provided by the other central detector components this results in an impact parameter resolution of $16 \mu \mathrm{m}$ for tracks in $\mathrm{Z}^{0} \rightarrow \mu^{+} \mu^{-}$and $\mathrm{Z}^{0} \rightarrow \mathrm{e}^{+} \mathrm{e}^{-}$events. The central detector is positioned inside a solenoidal coil that provides a uniform magnetic field of $0.435 \mathrm{~T}$. The momentum resolution obtained is approximately $\left(\sigma_{p_{x y}} / p_{x y}\right)^{2}=(0.02)^{2}+\left(0.0015 p_{x y}\right)^{2}$, where $p_{x y}$ is in GeV. In addition to tracking charged particles, the jet chamber provides measurements of the ionization loss, $\mathrm{d} E / \mathrm{d} x$, of charged particles, which are used for particle identification. The coil is surrounded by a time-of-flight counter array and a lead-glass electromagnetic calorimeter with presampler. Outside the electromagnetic calorimeter is the instrumented return yoke of the magnet, which forms the hadron calorimeter. This is surrounded by muon chambers.

\section{Particle Identification}

Charged pions and kaons are identified using $\mathrm{d} E / \mathrm{d} x$ information from the jet chamber [14]. For the momentum region between $2 \mathrm{GeV}$ and $20 \mathrm{GeV}$ the separation between pions and kaons is greater than two standard deviations. We consider a particle to be consistent with a specific hypothesis if the probability for the measured $\mathrm{d} E / \mathrm{d} x$ value is calculated to be greater than $1 \%$. For kaons, if the measured $\mathrm{d} E / \mathrm{d} x$ is higher than the expected value, we tighten this requirement to be greater than $3 \%$.

The electron identification procedure used in this analysis is similar to that described in a previous publication [15] and covers the angular range $|\cos \theta|<0.72$. It uses the $\mathrm{d} E / \mathrm{d} x$ measured in the jet chamber and the quantity $E_{\text {cone }} / p$, where $E_{\text {cone }}$ is the energy deposited in the calorimeter in a cone around the extrapolated position of the central detector track of momentum $p$. Both measured quantities are compared with the expected values for an electron hypothesis and are required to be within 2 standard deviations if they are lower. Furthermore, vertex information is used to reject electron candidates arising from photon conversions [16]. In the kinematic range relevant to this analysis the electron identification efficiency is about $80 \%$.

Muons are identified [16] by associating central detector tracks with track segments in the muon chambers, requiring a position match in two orthogonal coordinates. In addition, loose requirements on $\mathrm{d} E / \mathrm{d} x$ are made to reject kaons and protons. The average identification efficiency is approximately $80 \%$ for muons with $p>2 \mathrm{GeV}$ in the acceptance region relevant to this analysis.

\section{Monte Carlo Simulation}

In order to obtain the reconstruction efficiencies for each decay mode and study the background we used a full Monte Carlo simulation of the OPAL detector [17]. The JETSET 7.3 Monte Carlo program [18] was used to generate samples of semileptonic B decay events in each of the $\mathrm{D}^{(*)} \ell^{-}$channels. Decays to $\mathrm{P}$-wave charmed mesons were included in these simulations. Peterson parameterization [20] was used for the $b$ quark fragmentation, with $\epsilon_{b}=0.0057$, corresponding to $\left\langle x_{E}\right\rangle=0.697[15]$, where $\left\langle x_{E}\right\rangle=\left\langle E_{\text {Bhadron }}\right\rangle / E_{\text {beam }}$. All these events were then passed through a full detector simulation [17] from which we obtained the reconstruction efficiencies for such events in the OPAL detector. 
We chose to estimate the decay model systematic uncertainties by using the JETSET sample and allowing $f^{* *}$, the fraction of semileptonic B meson decays to any charmed meson other than the $\mathrm{D}$ or $\mathrm{D}^{*}$, to vary in the range $0.36 \pm 0.12$, as measured by CLEO [21] and $p_{v}$, the fraction of $\mathrm{P}$-wave charmed meson decays to a $\mathrm{D}^{*}$ meson, to vary in the range $0.54 \pm 0.30$, as assumed previously [19]. As well as the P-wave mesons $f^{* *}$ could include higher mass states or non-resonant decays. However, in our Monte Carlo simulation we assumed that the $f^{* *}$ component was saturated by the four $\mathrm{P}$-wave mesons, $\mathrm{D}_{J}$, and therefore included only them in the simulation.

In order to study the kinematics of the semileptonic decays to the narrow P-wave charmed mesons, additional high statistics samples of simulated events have been generated using a fast simulation of the OPAL detector [17]. For this study the masses of the $\mathrm{D}_{1}^{0}(2420), \mathrm{D}_{2}^{* 0}(2460), \mathrm{D}_{1}^{+}(2420)$ and $\mathrm{D}_{2}^{*+}(2460)$ states were set to the world average values [1], and were assumed to have widths of $20 \mathrm{MeV}$.

\section{$5 \quad \mathrm{D}^{0}, \mathrm{D}^{+}$and $\mathrm{D}^{*+}$ Meson Production}

\subsection{Event Selection}

The data used in this analysis include all the data recorded in 1992 and 1993, as well as those taken after the microvertex detector was commissioned in 1991. The data were collected from $\mathrm{e}^{+} \mathrm{e}^{-}$annihilations at centre of mass energies between 88.5 and $93.8 \mathrm{GeV}$. The selection criteria for hadronic $Z^{0}$ decays are described elsewhere [22] and have an efficiency of $(98.4 \pm 0.4) \%$, with negligible contamination. After data quality and detector performance requirements, the available data sample consists of 1.72 million events.

The selection of $\mathrm{B} \rightarrow \overline{\mathrm{D}} \ell^{+} \mathrm{X}$ and $\mathrm{B} \rightarrow \overline{\mathrm{D}}^{*} \ell^{+} \mathrm{X}$ events used kinematic and vertex information from the decays. In table 1 we summarize the selection criteria for the five different decay modes which we consider. The selection is similar to that used previously [19]. D mesons were selected by considering all track combinations consistent with the appropriate particle identification hypotheses. In addition, for the $\mathrm{D}^{0}$ and $\mathrm{D}^{+}$decays tighter requirements were placed on the kaon identification to reduce the misidentification background. The probability of a pion hypothesis was required to be smaller than $5 \%$ for the kaon candidates in these decays.

To reduce combinatorial background $\mathrm{D}$ and $\mathrm{D}^{*}$ meson candidates were required to have energy $\left(E_{\mathrm{D}^{(*)}}\right)$ greater than $5-8 \mathrm{GeV}$ depending on the decay mode. To select $\mathrm{D}^{*+}$ candidates we demanded a $\pi^{+}$in addition to a $\mathrm{D}^{0}$ candidate. The difference between the mass of the $D^{*+}$ candidate and that of the $D^{0}$ was required to be in the range $0.1415-$ $0.1485 \mathrm{GeV}$. For some decay modes we also cut on $\cos \theta^{*}$, where $\theta^{*}$ is the angle between the $\mathrm{K}^{-}$and the $\mathrm{D}$ boost direction in the $\mathrm{D}$ rest frame. The isotropic decay of the pseudoscalar D mesons has a flat distribution in $\cos \theta^{*}$, whereas, the background, particularly that resulting from particle misidentification, tends to be concentrated at large $\left|\cos \theta^{*}\right|$.

Lepton candidates were required to have $p>2 \mathrm{GeV}$. All $\mathrm{D}^{(*)} \ell^{-}$combinations were considered as possible $\mathrm{B}$ candidates. To suppress random combinations we required the mass $\left(m_{\mathrm{D}^{(*)} \ell}\right)$ and energy $\left(E_{\mathrm{D}^{(*)} \ell}\right)$ of the candidates to satisfy certain minimum criteria, shown in table 1 . In addition all candidates were required to have $m_{\mathrm{D}^{(*)} \ell}<5.35 \mathrm{GeV}$.

In order to reject poorly determined and possibly badly reconstructed decays we required the lepton track and at least two of the D decay tracks to have at least one associated microvertex hit. This ensured that vertex reconstruction was dominated by 


\begin{tabular}{|l|c|c|c|c|c|c|}
\hline Decay mode & $\begin{array}{c}E_{\mathrm{D}^{(*)}} \\
(\mathrm{GeV})\end{array}$ & $\cos \theta^{*}$ & $\begin{array}{c}m_{\mathrm{D}^{(*)} \ell} \\
(\mathrm{GeV})\end{array}$ & $\begin{array}{c}E_{\mathrm{D}^{(*)} \ell} \\
(\mathrm{GeV})\end{array}$ & $l_{\mathrm{D}} / \sigma_{l}$ & $l_{\mathrm{B}} / \sigma_{l}$ \\
\hline $\mathrm{D}^{+} \rightarrow \mathrm{K}^{-} \pi^{+} \pi^{+}$ & $>8$ & $<0.9$ & $>3.0$ & $>13.5$ & $>0$ & $>-1$ \\
\hline $\begin{array}{l}\mathrm{D}^{*+} \rightarrow \mathrm{D}^{0} \pi^{+} \\
\mathrm{D}^{0} \rightarrow \mathrm{K}^{-} \pi^{+}\end{array}$ & $>5$ & $\left|\cos \theta^{*}\right|<0.95$ & $>2.8$ & $>9.0$ & - & - \\
\hline $\begin{array}{l}\mathrm{D}^{*+} \rightarrow \mathrm{D}^{0} \pi^{+}, \\
\mathrm{D}^{0} \rightarrow \mathrm{K}^{-} \pi^{+} \pi^{+} \pi^{-}\end{array}$ & $>8$ & - & $>3.0$ & $>13.5$ & - & - \\
\hline $\mathrm{D}^{0} \rightarrow \mathrm{K}^{-} \pi^{+}$ & $>6$ & $<0.8$ & $>3.0$ & $>13.5$ & $>-1$ & $>-1$ \\
\hline $\mathrm{D}^{0} \rightarrow \mathrm{K}^{-} \pi^{+} \pi^{+} \pi^{-}$ & $>8$ & - & $>3.0$ & $>13.5$ & $>-1$ & $>0$ \\
\hline
\end{tabular}

Table 1: Decay mode dependent selection criteria.

tracks with microvertex detector information.

To reconstruct the decay vertices we minimized the $\chi^{2}$ for a vertex fit with respect to $\left(x_{B}, y_{B}, l_{D}, \kappa_{1} \ldots \kappa_{i}, \phi_{1} \ldots \phi_{i}\right)$, where $\left(x_{B}, y_{B}\right)$ are the coordinates of the $\mathrm{B}$ decay vertex, $l_{D}$ is the decay length of the D meson and $\kappa_{i}$ and $\phi_{i}$ are the curvature and angle of the $i^{\text {th }}$ track at the relevant decay vertex. The direction of flight of the $\mathrm{D}$ meson was fixed to correspond to its momentum vector in the fit. We demanded that the probability for the vertex fit, $\mathrm{P}\left(\chi_{\text {vertex }}^{2}\right)$, was greater than $1 \%$ in order to suppress random track combinations and badly reconstructed vertices. The $\mathrm{B}$ hadron decay length was calculated in the $r-\phi$ plane using the position of the reconstructed $\mathrm{D}^{(*)} \ell^{-}$vertex and the average $\mathrm{e}^{+} \mathrm{e}^{-}$interaction point. The average interaction point was determined using charged tracks selected from many consecutive events during that LEP fill. The B decay length was signed according to the cosine of the angle between the vector separating $\left(x_{B}, y_{B}\right)$ from the average interaction point and the $\mathrm{D}^{(*)} \ell^{-}$momentum vector. To convert the decay length into three dimensions we estimated $\sin \theta$ for the $\mathrm{B}$ hadron from the $\overline{\mathrm{D}}^{(*)} \ell^{+}$momentum vector. The $\mathrm{B}$ hadrons had typical decay lengths of $3 \mathrm{~mm}$ which were reconstructed with a resolution of about $300 \mu \mathrm{m}$. The $\mathrm{D}^{0}\left(\mathrm{D}^{+}\right)$mesons had typical decay lengths of $1 \mathrm{~mm}(2.5 \mathrm{~mm})$ which were reconstructed with resolutions of about $800 \mu \mathrm{m}$. For the inclusive $\mathrm{D}^{0}$ and $\mathrm{D}^{+}$samples we applied loose selection criteria to both the $\mathrm{B}$ and $\mathrm{D}$ decay lengths in order to further reduce the combinatorial background.

The D mass distributions for the five different decay modes are shown in figures 1 to 3 . A signal is visible in each of these decay modes. The $\mathrm{K}^{-} \pi^{+}$mass distributions also show a (satellite) peak around $1.6 \mathrm{GeV}$. An enhancement is expected in this region from the partially reconstructed decays, particularly $\mathrm{D}^{0} \rightarrow \mathrm{K}^{-} \rho^{+}, \rho^{+} \rightarrow \pi^{+} \pi^{0}$, in which the $\pi^{0}$ is not reconstructed. These decays were not used for the measurement of $\mathrm{D}^{0}, \mathrm{D}^{+}$and $\mathrm{D}^{*+}$ meson production but were used in the $\mathrm{D}^{*+}$ channel for the $\mathrm{P}$-wave meson search. We determined the signal and background in each channel by fitting the mass distributions using a function consisting of a sum of a Gaussian and a second order polynomial. The 
masses and widths of the signal Gaussians are allowed to vary in the fit. The fitted D meson masses are consistent with the accepted values [1]. The enhancements around $1.6 \mathrm{GeV}$ are also parameterized as Gaussians and included in the fit. The background levels in the signal regions are found to be insensitive to this parameterization.

\subsection{Results}

To be confident of the efficiencies calculated using the Monte Carlo simulations, we compared the most sensitive kinematic distributions, $E_{\mathrm{D}^{(*)} \ell}$ and $m_{\mathrm{D}^{(*)} \ell}$ with similar distributions produced using the data (see figures 4 and 5). We also compared these Monte Carlo distributions with those produced using the ISGW model [2] for semileptonic B decays. In both cases, agreement was shown between the distributions, the resulting model uncertainties being smaller than those due to $f^{* *}$ and $p_{v}$.

In addition to the $b \rightarrow \mathrm{D}^{(*)} \ell^{-} \mathrm{X}$ decays there are a number of other possible sources of $\mathrm{D}^{(*)} \ell^{-}$combinations which may contribute to the observed signals. The rates of these background processes were determined and subtracted from the observed signals in order to determine the $b \rightarrow \mathrm{D}^{(*)} \ell^{-} \mathrm{X}$ rates.

To quantify the background due to $D^{(*)}$ mesons combined with fake leptons we fitted the $\mathrm{D}^{(*)} \ell^{+}$combination mass distributions in the same way as we fitted the signal. Because of the requirement that $m_{\mathrm{D}^{(*)} \ell}<5.35 \mathrm{GeV}$ combinations of $\mathrm{D}^{(*)}$ mesons and leptons from different $b$ hadrons are effectively excluded. For each channel the fit was consistent with zero and no correction was made.

Two other sources of background were considered, $\mathrm{B} \rightarrow \mathrm{D}_{s}^{(*)} \overline{\mathrm{D}}^{(*)}$ where the $\mathrm{D}_{s}^{(*)}$ decay includes a lepton, and $\mathrm{B} \rightarrow \mathrm{D}^{(*)} \tau \mathrm{X}$ where the $\tau$ decays to either an electron or muon. We used full Monte Carlo simulations of these backgrounds to obtain the reconstruction efficiencies and using the previously measured branching ratios, $B\left(\mathrm{~B} \rightarrow \mathrm{D}_{s}^{(*)} \overline{\mathrm{D}}^{(*)}\right)=5.0 \pm$ $0.9 \%$ [1] and $B(b \rightarrow \tau \mathrm{X})=4.1 \pm 1.0 \%$ [1]. We quantified these background contributions to be between 2 and $5 \%$ of the samples and subtracted them accordingly. The uncertainty in this calculation is included as a contribution to the systematic error.

From the background subtracted number of reconstructed data events in each channel and the reconstruction efficiencies calculated using the Monte Carlo simulations the total number of events for each channel in the data sample was estimated. This was done independently for the electron and muon events, and for each channel the number of electron and muon events were consistent with each other.

The number of $\mathrm{Z}^{0} \rightarrow b \bar{b}$ decays in our data sample was calculated using the OPAL value of $\Gamma_{b \bar{b}} / \Gamma_{h a d}=0.2171 \pm 0.0030$ [23]. The error on $\Gamma_{b \bar{b}} / \Gamma_{\text {had }}$ is considered as a systematic uncertainty. From this and using the ratio, $R_{42}=B\left(\mathrm{D}^{0} \rightarrow \mathrm{K}^{-} \pi^{+} \pi^{-} \pi^{+}\right) / B\left(\mathrm{D}^{0} \rightarrow\right.$ $\left.\mathrm{K}^{-} \pi^{+}\right)=2.01 \pm 0.13[1]$ to normalize the $\mathrm{D}^{0} \rightarrow \mathrm{K} 3 \pi$ measurements to the $\mathrm{D}^{0} \rightarrow \mathrm{K} \pi$ results, we obtained the product branching ratios for each decay mode. The measurement uncertainty on $R_{42}$ was considered as a systematic error. Taking the weighted mean of the product branching ratios for the two $\mathrm{D}^{0}$ decay modes and averaging over the electron and muon channels the following results were obtained:

$$
\begin{aligned}
B\left(b \rightarrow \mathrm{D}^{+} \ell X\right) \cdot B\left(\mathrm{D}^{+} \rightarrow \mathrm{K}^{-} \pi^{+} \pi^{+}\right) & =(1.82 \pm 0.20) \times 10^{-3} \\
B\left(b \rightarrow \mathrm{D}^{0} \ell X\right) \cdot B\left(\mathrm{D}^{0} \rightarrow \mathrm{K}^{-} \pi^{+}\right) & =(2.52 \pm 0.14) \times 10^{-3} \\
B\left(b \rightarrow \mathrm{D}^{*+} \ell X\right) \cdot B\left(\mathrm{D}^{*+} \rightarrow \mathrm{D}^{0} \pi^{+}\right) \cdot B\left(\mathrm{D}^{0} \rightarrow \mathrm{K}^{-} \pi^{+}\right) & =(7.53 \pm 0.47) \times 10^{-4}
\end{aligned}
$$




\subsection{Systematic Uncertainties for the Product Branching Ratios}

As discussed in section 4 there are significant uncertainties in the reconstruction efficiencies due to the modelling and decay rates of the different $\mathrm{D}_{J}$ decays. To estimate this uncertainty we varied the parameters $f^{* *}$ and $p_{v}$ by \pm 0.12 and \pm 0.30 respectively, and used the individual reconstruction efficiencies for $\mathrm{B} \rightarrow \mathrm{D} \ell \mathrm{X}, \mathrm{B} \rightarrow \mathrm{D}^{*} \ell \mathrm{X}$ and $\mathrm{B} \rightarrow \mathrm{D}_{J} \ell \mathrm{X}$ from the Monte Carlo simulation to calculate the model systematic uncertainties.

To evaluate the uncertainty due to the $b$ quark fragmentation we have studied samples of Monte Carlo data, made using a fast simulation of the OPAL detector, and varied the Peterson parameterization [20], $\epsilon_{b}$, between 0.0025 and 0.0095 . This gives a variation in $\left\langle x_{E}\right\rangle$ for $\mathrm{B}$ hadrons consistent with the OPAL average result [15]. The systematic uncertainty from this variation in the reconstruction efficiency, averaged over all channels, was found to be $2.9 \pm 1.4 \%$.

\begin{tabular}{|c|c|c|c|}
\hline $\begin{array}{l}\text { Systematic } \\
\text { source }\end{array}$ & $\left(b \rightarrow \underset{\%}{\left.\mathrm{D}^{+} \ell \mathrm{X}\right)}\right.$ & $\left(b \rightarrow \underset{\%}{\mathrm{D}^{0} \ell \mathrm{X}}\right)$ & $\left(b \rightarrow \mathrm{D}_{\%}^{*+} \ell \mathrm{X}\right)$ \\
\hline$f^{* *} \pm 0.12$ & 3.1 & 2.6 & 2.9 \\
\hline$p_{v} \pm 0.30$ & 1.9 & 0.9 & 3.4 \\
\hline$b$ fragmentation & 2.9 & 2.9 & 2.9 \\
\hline Monte Carlo statistics & 1.6 & 1.6 & 2.3 \\
\hline Background subtraction & 1.9 & 1.4 & 1.8 \\
\hline$e \mathrm{ID}$ & 2.8 & 2.8 & 2.8 \\
\hline$\mu \mathrm{ID}$ & 1.1 & 1.1 & 1.1 \\
\hline Si matching efficiency & 1.1 & 2.0 & 1.1 \\
\hline$d E / d x($ Kaon ID) & 3.3 & 3.3 & 2.7 \\
\hline Detector resolution & 0.3 & 1.2 & - \\
\hline$\Gamma_{b \bar{b}} / \Gamma_{h a d}$ & 1.4 & 1.4 & 1.4 \\
\hline$R_{24}$ & - & 1.9 & 1.8 \\
\hline Total & 6.8 & 6.9 & 7.4 \\
\hline
\end{tabular}

Table 2: Summary of systematic uncertainties on the three different product branching ratio measurements.

The systematic uncertainties due to the electron and muon identification in OPAL have been calculated in a similar way to previous publications $[15,16]$. The good agreement between the branching ratio measurements obtained individually with electrons and muons provides additional confirmation that these errors are understood. In calculating the total systematic error the data-weighted mean of these two uncertainties was used.

In order to estimate the systematic uncertainties in the efficiencies for both the $\mathrm{d} E / \mathrm{d} x$ selection criteria and the silicon microvertex detector matching efficiency we studied inclusive samples of $\mathrm{D}^{*+} \rightarrow\left(\mathrm{D}^{0}\right) \pi^{+}, \mathrm{D}^{0} \rightarrow \mathrm{K}^{-} \pi^{+}$events. The selection efficiencies were determined by fitting the $\mathrm{D}^{0}$ mass peak before and after applying the selection criteria. These efficiencies were then compared directly with fully simulated Monte Carlo event samples which pass identical selection criteria. These studies indicate excellent agreement between data and the Monte Carlo simulation in the case of both the $\mathrm{d} E / \mathrm{d} x$ modelling and the silicon matching efficiencies. As a result of this study the estimated systematic uncertainty on the loose $\mathrm{d} E / \mathrm{d} x$ selection (see section 3 ) was estimated to be $2.1 \%$, whilst with the addition of the cut on the pion probability for the kaon candidates the error increased to $3.1 \%$. Likewise the silicon matching efficiency for single tracks was 
estimated to have a relative uncertainty of $1.0 \%$. The combined errors were determined according to the statistical weights of the different decay modes to obtain the total systematic error contributions. To account for detector resolution systematic effects on the loose cuts on the B and D decay lengths, we varied the resolutions by $\pm 10 \%$ and obtained the corresponding variation in the reconstruction efficiencies.

Combining all the systematic errors in quadrature we obtain:

$$
\begin{aligned}
B\left(b \rightarrow \mathrm{D}^{+} \ell X\right) \cdot B\left(\mathrm{D}^{+} \rightarrow \mathrm{K}^{-} \pi^{+} \pi^{+}\right) & =(1.82 \pm 0.20 \pm 0.12) \times 10^{-3} \\
B\left(b \rightarrow \mathrm{D}^{0} \ell X\right) \cdot B\left(\mathrm{D}^{0} \rightarrow \mathrm{K}^{-} \pi^{+}\right) & =(2.52 \pm 0.14 \pm 0.17) \times 10^{-3} \\
B\left(b \rightarrow \mathrm{D}^{*+} \ell X\right) \cdot B\left(\mathrm{D}^{*+} \rightarrow \mathrm{D}^{0} \pi^{+}\right) \cdot B\left(\mathrm{D}^{0} \rightarrow \mathrm{K}^{-} \pi^{+}\right) & =(7.53 \pm 0.47 \pm 0.56) \times 10^{-4} .
\end{aligned}
$$

It should be noted that all the systematic errors, other than those due to Monte Carlo statistics, are correlated between the different samples.

\subsection{Discussion}

These results may be compared with those from CLEO [21] $]^{3}$

$$
\begin{aligned}
B\left(\mathrm{~B} \rightarrow \mathrm{D}^{+} \ell X\right) \cdot B\left(\mathrm{D}^{+} \rightarrow \mathrm{K}^{-} \pi^{+} \pi^{+}\right) & =(2.46 \pm 0.55) \times 10^{-3}, \\
B\left(\mathrm{~B} \rightarrow \mathrm{D}^{0} \ell X\right) \cdot B\left(\mathrm{D}^{0} \rightarrow \mathrm{K}^{-} \pi^{+}\right) & =(2.90 \pm 0.38) \times 10^{-3} .
\end{aligned}
$$

At the $\Upsilon(4 S)$ only $\mathrm{B}^{0}$ and $\mathrm{B}^{+}$mesons are produced. A comparison of the ratio of the CLEO measurement with the results presented here for the $\mathrm{D}^{+}$and $\mathrm{D}^{0}$ channels makes it possible to determine the fraction of $b$ quarks which fragment to $\mathrm{B}^{0}$ and $\mathrm{B}^{+}$mesons. In order to do this it was necessary to estimate the contribution to the rates from $\mathrm{B}_{s}$ and $b$-baryons. The semileptonic decays of $b$-baryons are expected to be dominated by decay modes involving charm baryons and are unlikely to contribute to the $\mathrm{D}^{(*)} \ell$ signals. However $\mathrm{B}_{s}$ mesons may decay via the chain $\mathrm{B}_{s} \rightarrow \mathrm{D}_{s .}^{-} \ell^{+} \mathrm{X}, \mathrm{D}_{s . J} \rightarrow \mathrm{D}^{(*)} \mathrm{K}$. Using our previously published measurement of the $\mathrm{B}_{s} \rightarrow \mathrm{D}_{s}^{-} \ell^{+} \mathrm{X}$ branching ratio [24], and assuming that $f^{* *}=0.36 \pm 0.12$ holds for $\mathrm{B}_{s}$ decays, we estimated there to be $2 \pm 1 \% \mathrm{~B}_{s}$ in our samples. After correcting for this and assuming that the $\mathrm{B}^{+} / \mathrm{B}^{0}$ ratio is the same in $Z^{0} \rightarrow b \bar{b}$ events as in $\Upsilon(4 S)$ decays we obtain:

$$
f\left(b \rightarrow \mathrm{B}^{0}\right)+f\left(b \rightarrow \mathrm{B}^{+}\right)=0.81 \pm 0.07 \pm 0.09
$$

where the first error was due to the error on our results and the second due to those on the CLEO results.

It is also of interest to calculate the total charm meson production rate in semileptonic $\mathrm{B}$ decays. In order to do this we used the following $\mathrm{D}$ branching ratios $[1]: B\left(\mathrm{D}^{+} \rightarrow\right.$ $\left.\mathrm{K}^{-} \pi^{+} \pi^{+}\right)=0.091 \pm 0.006, B\left(\mathrm{D}^{0} \rightarrow \mathrm{K}^{-} \pi^{+}\right)=0.0401 \pm 0.0014$ and $B\left(\mathrm{D}^{*+} \rightarrow \mathrm{D}^{0} \pi^{+}\right)=$ $0.681 \pm 0.013$, to obtain the inclusive branching ratios:

$$
\begin{aligned}
B\left(b \rightarrow \mathrm{D}^{+} \ell X\right) & =(2.00 \pm 0.22 \pm 0.13 \pm 0.13) \% \\
B\left(b \rightarrow \mathrm{D}^{0} \ell X\right) & =(6.28 \pm 0.35 \pm 0.42 \pm 0.22) \% \\
B\left(b \rightarrow \mathrm{D}^{*+} \ell X\right) & =(2.76 \pm 0.17 \pm 0.21 \pm 0.11) \%
\end{aligned}
$$

where the third source of error is due to the error on the $\mathrm{D}$ and $\mathrm{D}^{*}$ branching ratios.

\footnotetext{
${ }^{3}$ We have multiplied the branching ratios quoted in reference [21] by the D branching ratios used to obtain these values. The errors on these product branching ratios are assumed to be uncorrelated.
} 
The total inclusive semileptonic branching ratio to modes containing a $\mathrm{D}^{0}$ or $\mathrm{D}^{+}$meson is therefore $(8.28 \pm 0.41 \pm 0.57 \pm 0.25) \%$, taking into account the correlated systematic errors. This can be compared with our measurement of the total $b$ semileptonic branching ratio of $10.5 \pm 0.6 \pm 0.5 \%[15]$.

\section{Search for P-wave Charmed Mesons}

\subsection{Introduction}

The P-wave charmed mesons are expected to consist of four different charged and neutral states with spin-parity $\left(0^{+}, 1^{+}, 1^{+}, 2^{+}\right)$. Two of these states, with spin-parity $\left(1^{+}, 2^{+}\right)$are expected to be narrow ${ }^{4}$ with natural widths of about $20 \mathrm{MeV}$. The others are expected to be broad, with widths of hundreds of $\mathrm{MeV}$ [25]. Experimental evidence exists for only the narrow $\mathrm{P}$-wave charmed meson states. The two neutral mesons $\mathrm{D}_{1}^{0}(2420)$ and $\mathrm{D}_{2}^{* 0}(2460)$ are now experimentally well established $[5,6,8]$. The spin assignment is based on both the observed angular distribution of the decay products and the fact that the decay $\mathrm{D}_{1}^{0}(2420) \rightarrow \mathrm{D}^{+} \pi^{-}$, which is forbidden by spin-parity, has not been observed. The average of the measured ratio of decay modes of the $D_{2}^{* 0}(2460)$ meson $[1,8]$ :

$$
\frac{B\left(\mathrm{D}_{2}^{* 0}(2460) \rightarrow \mathrm{D}^{+} \pi^{-}\right)}{B\left(\mathrm{D}_{2}^{* 0}(2460) \rightarrow \mathrm{D}^{*+} \pi^{-}\right)}=2.3 \pm 0.6
$$

is also in good agreement with expectations for the tensor state [25]. Recent experimental evidence has established the existence of the corresponding charged states $\mathrm{D}_{1}^{+}(2420)$ and $\mathrm{D}_{2}^{*+}(2460)[6,7,9]$.

Using a combination of the known $\mathrm{D}^{*}$ branching ratios, the isospin predictions given by:

$$
\begin{aligned}
& B\left(\mathrm{D}_{J}^{+} \rightarrow \mathrm{D}^{(*) 0} \pi^{+}\right)=2 B\left(\mathrm{D}_{J}^{+} \rightarrow \mathrm{D}^{(*)+} \pi^{0}\right), \\
& B\left(\mathrm{D}_{J}^{0} \rightarrow \mathrm{D}^{(*)+} \pi^{-}\right)=2 B\left(\mathrm{D}_{J}^{0} \rightarrow \mathrm{D}^{(*)} \pi^{0}\right),
\end{aligned}
$$

and the measured ratio of decay modes for the $\mathrm{D}_{2}^{* 0}(2460)$ meson in equation 1 it is possible to estimate the decay modes of the $\mathrm{D}_{J}$ mesons to different $\mathrm{D}^{(*)} \pi$ final states. To do this we assumed these decay modes saturate the $\mathrm{D}_{J}$ decays and assumed that the $\mathrm{D}_{2}^{*+}(2460)$ decays to $\mathrm{D} \pi / \mathrm{D}^{*} \pi$ in a similar ratio to that given by equation 1 . These predictions are listed in table 3 . The expected mean values of $\Delta_{m}=m\left(\mathrm{D}^{(*)} \pi\right)-m\left(\mathrm{D}^{(*)}\right)$ are also shown. As with the familar $\mathrm{D}^{*+} \rightarrow \mathrm{D}^{0} \pi^{+}$decays, $\Delta_{m}$, calculated using the reconstructed $\mathrm{D}^{(*)}$ masses, has a better experimental resolution than the raw $m\left(\mathrm{D}^{(*)} \pi\right)$ distribution.

Samples of simulated P-wave charmed mesons have been used to study the expected signals in the OPAL detector. Because the pseudovector and tensor states are almost degenerate a mixture of the states is found to result in a broad enhancement of $\sim 35 \mathrm{MeV}$ width around $\Delta_{m} \sim 440 \mathrm{MeV}$ in $\mathrm{D}^{0} \pi^{+}, \mathrm{D}^{+} \pi^{-}$and $\mathrm{D}^{*+} \pi^{-}$distributions. This is as a result of the merging of the two individual peaks of width $\sim 25 \mathrm{MeV}$. Due to the low q-value of the $\mathrm{D}^{*}$ decay, the partially reconstructed decays of the type $\mathrm{D}_{J} \rightarrow \mathrm{D}^{*} \pi, \mathrm{D}^{*} \rightarrow \mathrm{DX}$, where $\mathrm{X}$ is a $\pi^{0}$ or $\gamma$ form a peak with a slightly degraded mass resolution. In addition both the simulated $\mathrm{D}^{0} \pi^{+}$and $\mathrm{D}^{+} \pi^{-} \Delta_{m}$ distributions contain a second narrow peak at $\sim 590 \mathrm{MeV}$ due to the direct decay of the tensor mesons to $\mathrm{D} \pi$ final states. It was concluded from

\footnotetext{
${ }^{4}$ Assuming mixing between the two $1^{+}$states is small.
} 


\begin{tabular}{|c|c|c|c|c|c|}
\hline Decay Mode & B.R. & $\begin{array}{c}\Delta_{m} \\
(\mathrm{MeV})\end{array}$ & Decay Mode & B.R. & $\begin{array}{c}\Delta_{m} \\
(\mathrm{MeV})\end{array}$ \\
\hline $\begin{aligned} \mathrm{D}_{2}^{* 0}(2460) \rightarrow & \underline{\mathrm{D}}^{+} \underline{\pi}^{-} \\
\mathrm{D}_{2}^{* 0}(2460) \rightarrow & \mathrm{D}^{*+} \underline{\pi}^{-} \\
& \hookrightarrow \underline{\mathrm{D}}^{+} \mathrm{X} \\
\mathrm{D}_{2}^{* 0}(2460) \rightarrow & \mathrm{D}^{*+} \pi^{-} \\
& \hookrightarrow \underline{\mathrm{D}}^{0} \underline{\pi}^{+}\end{aligned}$ & $46 \%$ & $448 \pm 2$ & $\begin{aligned} \mathrm{D}_{2}^{*+}(2460) & \rightarrow \underline{\mathrm{D}}^{0} \pi^{+} \\
\mathrm{D}_{2}^{*+}(2460) & \rightarrow \mathrm{D}^{* 0} \frac{\pi}{}^{+} \\
& \hookrightarrow \underline{\mathrm{D}}^{0} \mathrm{X}\end{aligned}$ & $46 \%$ & $591 \pm 6$ \\
\hline $\begin{aligned} \mathrm{D}_{1}^{0}(2420) \rightarrow & \mathrm{D}^{*+} \underline{\pi}^{-} \\
& \hookrightarrow \underline{\mathrm{D}}^{+} \mathrm{X} \\
\mathrm{D}_{1}^{0}(2420) \rightarrow & \mathrm{D}^{*+} \underline{\pi}^{-} \\
& \hookrightarrow \underline{\mathrm{D}}^{0} \underline{\pi}^{+}\end{aligned}$ & $44 \%$ & $413 \pm 3$ & $\begin{aligned} \mathrm{D}_{1}^{+}(2420) \rightarrow & \mathrm{D}^{* 0} \frac{\pi}{}^{+} \\
& \hookrightarrow \underline{\mathrm{D}}^{0} \mathrm{X}\end{aligned}$ & $67 \%$ & $436 \pm 9$ \\
\hline
\end{tabular}

Table 3: A summary of $\mathrm{D}_{J}$ decay modes to which this analysis is sensitive with the expected branching ratio and $\Delta_{m}$ for each channel. Detected particles are underlined.

this study that while it is experimentally challenging to resolve directly the $\Delta_{m}$ peaks at $\sim 440 \mathrm{MeV}$ due to a mixture of decays, the observation of the tensor meson decays at $\Delta_{m} \sim 590 \mathrm{MeV}$ provides a clear signature for these states which then could be used to estimate the contributions from the tensor mesons to the unresolved peaks.

\subsection{Event Selection}

To search for the presence of $\mathrm{P}$-wave mesons we used the event samples of inclusive $b \rightarrow \mathrm{D}^{(*)} \ell^{-} \mathrm{X}$ decays obtained using the selection described in section 5.1. To reduce the combinatorial background in these samples, slightly tighter selection criteria were applied. Events which passed these selection criteria were also required to be in a mass region within approximately 2 standard deviations of the $\mathrm{D}$ meson mass. These additional selection criteria are listed in table 4 . In order to exclude $\mathrm{D}^{*+} \rightarrow \mathrm{D}^{0} \pi^{+}$decays from the $b \rightarrow \mathrm{D}^{0} \ell^{-} \mathrm{X}$ samples, events were rejected if they contained any pion candidates satisfying $m\left(\mathrm{D}^{0} \pi^{+}\right)-m\left(\mathrm{D}^{0}\right)<0.16$. This excluded approximately $89 \%$ of the $\mathrm{D}^{*+}$ decays. To improve the available statistics, candidate $b \rightarrow \mathrm{D}^{*+} \ell^{-} \mathrm{X}$ decays were also selected from the satellite mass peak region $1.54-1.70 \mathrm{GeV}$ in the $\mathrm{D}^{*+} \rightarrow\left(\mathrm{K}^{-} \pi^{+}\right) \pi^{+}$distribution. Simulations indicate that these events are largely due to the decay $\mathrm{D}^{*+} \rightarrow\left(\mathrm{K}^{-} \rho^{+}\right) \pi^{+}$, in which the $\pi^{0}$ from the $\rho^{+}$decay is not reconstructed and for these decays the width of the $\Delta_{m}$ distribution due to a mixture of $\mathrm{D}_{1}^{0}(2420)$ and $\mathrm{D}_{2}^{* 0}(2460)$ decays is about $40 \mathrm{MeV}$, compared with $35 \mathrm{MeV}$ for fully reconstructed decays.

To search for the P-wave mesons we looked for additional pion candidates consistent with the kinematics of semileptonic $b$ hadron decays. The pion candidates were required to have a $\mathrm{d} E / \mathrm{d} x$ probability greater than $5 \%$ and the invariant mass of the $\mathrm{D}_{J} \ell^{-}$system was required to be less than $5.0 \mathrm{GeV}$. The isolation of signals of the $\mathrm{P}$-wave mesons is made difficult by the presence of many soft tracks not related to the $\mathrm{B}$ meson decay. In order to understand how to reduce this combinatorial background we studied the Monte Carlo samples of inclusive $b \rightarrow \mathrm{D} \ell X$ events. By comparing the distributions from random combinations of $\mathrm{D}^{(*)}$ mesons with fragmentation tracks, with those from the expected signal it was found that both the decay kinematics and the decay vertex topology provide good discrimination. The two most important selection criteria are the momentum of the 


\begin{tabular}{|l|c|c|c|c|c|}
\hline Decay mode & $l_{\mathrm{B}} / \sigma$ & $\mathrm{P}\left(\chi_{\text {vertex }}^{2}\right)$ & $\begin{array}{c}m(\mathrm{D}) \text { mass region } \\
(\mathrm{GeV})\end{array}$ & $\begin{array}{c}p(\pi) \\
(\mathrm{GeV})\end{array}$ & $\Delta_{d}$ \\
\hline $\mathrm{D}^{+} \rightarrow \mathrm{K}^{-} \pi^{+} \pi^{+}$ & $>0$ & $>0.1$ & $1.79-1.94$ & $>1.5$ & $>0.5$ \\
\hline $\mathrm{D}^{*+} \rightarrow\left(\mathrm{K}^{-} \pi^{+}\right) \pi^{+}$ & - & - & $1.79-1.94$ & $>1.0$ & $>0.5$ \\
$\mathrm{D}^{*+} \rightarrow\left(\mathrm{K}^{-} \pi^{+} \mathrm{X}\right) \pi^{+}$ & - & $>0.1$ & $1.540-1.700$ & $>1.0$ & $>0.5$ \\
$\mathrm{D}^{*+} \rightarrow\left(\mathrm{K}^{-} \pi^{+} \pi^{+} \pi^{-}\right) \pi^{+}$ & $>0.5$ & $>0.1$ & $1.815-1.915$ & $>1.5$ & $>0.5$ \\
\hline $\mathrm{D}^{0} \rightarrow \mathrm{K}^{-} \pi^{+}$ & $>0.5$ & - & $1.79-1.94$ & $>1.0$ & $>0.0$ \\
$\mathrm{D}^{0} \rightarrow \mathrm{K}^{-} \pi^{+} \pi^{+} \pi^{-}$ & $>0.5$ & $>0.1$ & $1.815-1.915$ & $>1.5$ & $>0.5$ \\
\hline
\end{tabular}

Table 4: Additional selection criteria applied to $b \rightarrow \mathrm{D}^{(*)} \ell \mathrm{X}$ samples and decay mode specific selection criteria used in $b \rightarrow \mathrm{D}_{J} \ell \mathrm{X}$ search.

transition pion candidate $(p(\pi))$, and

$$
\Delta_{d}=\left|d_{b m} / \sigma_{d_{b m}}\right|-\left|d_{v x} / \sigma_{d_{v x}}\right|
$$

where $d_{b m}\left(d_{v x}\right)$ is the $r-\phi$ impact parameter of the candidate transition pion with respect to the average beamspot (reconstructed $D^{(*)} \ell$ vertex) and $\sigma_{d_{b m}}\left(\sigma_{d_{v x}}\right)$ its error. The latter discriminator uses the fact that the background tracks arise from the primary vertex, whilst the signal pions originate from the $\mathrm{B}$ hadron decay vertex. In addition, to ensure that the pion candidate is consistent with the $\mathrm{B}$ decay vertex it was required that $\left|d_{v x} / \sigma_{d_{v x}}\right|<2.0$. The selection criteria listed in table 4 are based on these Monte Carlo studies. For the decay chains in which several $\mathrm{D}^{0}$ decay modes are used the selection criteria were chosen in order to obtain a similar signal-to-background ratio for the different modes. Figure 6 shows the overall effect of applying these criteria to a sample of simulated $b \rightarrow \mathrm{D}^{0} \ell^{-} \mathrm{X}$ events. The background was substantially reduced, whilst the $\mathrm{D}_{J}^{+}$signals were retained with about $50 \%$ efficiency. The other decay chains are similar. It can also be seen that in the mass region of the $\mathrm{D}_{J}^{+}$signals the combinatorial background is well described by an exponential function and it has been verified that the signals are accurately determined by fitting the $\Delta_{m}$ distribution with a function consisting of the linear combination of Gaussians together with an exponential background term.

In figure 7 the results of applying the $\mathrm{D}_{J}$ selection criteria to the data are shown. In each case a significant signal was observed close to that expected for the $\mathrm{D}_{J}$ decays. For the $\mathrm{D}^{*+} \pi^{-}$and $\mathrm{D}^{0} \pi^{+}$combinations the signals are not restricted to a single $\mathrm{D}^{0}$ decay mode and are therefore insensitive to changes to any specific selection requirements. In order to verify that these signals were not the result of a kinematic bias in the selection procedure we have studied two further control samples, shown in figures 8 and 9 . The first consists of $\mathrm{D}^{(*)} \pi$ combinations selected with identical kinematic criteria, but having $\Delta_{d}<-0.5$. In addition no requirement is placed on $\left|d_{v x} / \sigma_{v x}\right|$. These samples were expected to be depleted of $\mathrm{D}_{J}$ events and therefore were expected to provide a good description of the background due to random combinations with fragmentation tracks. The second sample studied consisted of events in which a combination of a $\mathrm{D}^{(*)}$ with a pion of the wrong charge passes all the selection criteria. In both cases the $\Delta_{m}$ distributions had no peaks corresponding to the observed signals, and were well described by an exponential function in the kinematic region of interest.

For the $\mathrm{D}^{0} \pi^{+}$combinations both the background normalization and exponential de- 
cay parameters were allowed to vary in the fit. However, for the $\mathrm{D}^{+} \pi^{-}$and $\mathrm{D}^{*+} \pi^{-}$ combinations, the background levels were low, so the exponential decay parameters were constrained to the values observed in the samples with $\Delta_{d}<-0.5$. In all cases the means and heights of the signal Gaussians were allowed to vary in the fit. The widths of the Gaussians were estimated from the results of the study discussed in section 6.1. However, variations of $\pm 10 \mathrm{MeV}$ were considered, in order to allow for differences in the $\mathrm{D}_{J}$ signal compositions and uncertainties in the natural widths of the different $\mathrm{D}_{J}$ states. These variations were considered as systematic errors. As a consistency check the fits were repeated using a second order polynomial to describe the background. This made no significant difference to the fitted number of signal events. Table 5 summarizes the fit results. In total a combined signal of $44 \pm 8_{-7}^{+3} \mathrm{D}_{J}^{0}$ and $48 \pm 10_{-6}^{+3} \mathrm{D}_{J}^{+}$events were observed, providing convincing evidence for the production of the $\mathrm{P}$-wave states in semileptonic $\mathrm{B}$ hadron decays.

\begin{tabular}{|l|c|c|c|c|c|}
\hline Sample & $\begin{array}{c}\sigma_{m} \\
(\mathrm{MeV})\end{array}$ & No. Events & $\begin{array}{c}\text { Fitted } \Delta_{m} \\
(\mathrm{MeV})\end{array}$ & Expected Signal & $\begin{array}{c}\text { Expected } \Delta_{m} \\
(\mathrm{MeV})\end{array}$ \\
\hline $\mathrm{D}^{+} \pi^{-}$ & $30 \pm 10$ & $17.1 \pm 4.8_{-5.2}^{+1.1}$ & $430 \pm 12 \pm 7$ & $\mathrm{D}_{1}^{0}(2420) / \mathrm{D}_{2}^{* 0}(2460)$ & $413-448$ \\
$\mathrm{D}^{+} \pi^{-}$ & $20 \pm 10$ & $7.0 \pm 2.9_{-0.8}^{+0.5}$ & $594 \pm 9_{-5}^{+6}$ & $\mathrm{D}_{2}^{* 0}(2460)$ & $589 \pm 2$ \\
\hline $\mathrm{D}^{*+} \pi^{-}$ & $35 \pm 10$ & $20.1 \pm 5.5_{-5.0}^{+3.0}$ & $439 \pm 14_{-11}^{+7}$ & $\mathrm{D}_{1}^{0}(2420) / \mathrm{D}_{2}^{* 0}(2460)$ & $413-448$ \\
\hline $\mathrm{D}^{0} \pi^{+}$ & $25 \pm 10$ & $30.1 \pm 8.0_{-3.1}^{+2.4}$ & $453 \pm 7 \pm 4$ & $\mathrm{D}_{1}^{+}(2420) / \mathrm{D}_{2}^{*+}(2460)$ & $436-449$ \\
$\mathrm{D}^{0} \pi^{+}$ & $20 \pm 10$ & $18.3 \pm 5.5_{-4.6}^{+1.5}$ & $567 \pm 8 \pm 6$ & $\mathrm{D}_{2}^{*+}(2460)$ & $591 \pm 6$ \\
\hline
\end{tabular}

Table 5: Summary of fit results to data distributions.

The signals for the neutral $\mathrm{D}_{J}$ mesons are in agreement with those expected from a mixture of $\mathrm{D}_{1}^{0}(2420)$ and $\mathrm{D}_{2}^{* 0}(2460)$ decays. However, the signals in the $\mathrm{D}_{J}^{+} \rightarrow \mathrm{D}^{0} \pi^{+}$ combinations agree less well. In particular the fitted value of $\Delta_{m}$ for the higher peak is about 2 standard deviations below the average value [1]. However, both signals are of about 3 standard deviations significance, and constraining the fit to the higher peak to $\Delta_{m}=591 \mathrm{MeV}$ gives a signal of $11.4 \pm 5.9$ events, which is consistent with our result. So in analogy with the neutral decays it seems natural to identify these signals with a mixture of $\mathrm{D}_{1}^{+}(2420) / \mathrm{D}_{2}^{*+}(2460)$ production.

\subsection{Efficiency calculation}

The efficiencies for reconstructing the different $b \rightarrow \mathrm{D}_{J} \ell^{-} \mathrm{X}$ decay modes were determined using similar Monte Carlo event samples to those described in section 4 . These Monte Carlo samples assumed equal production of the pseudovector and tensor states and because of the limited statistics available, the reconstruction efficiencies (excluding branching ratios) were averaged over states decaying to a particular $\mathrm{D}^{(*)} \pi \mathrm{X}$ combination. The $\mathrm{D}_{J}$ decays were simulated with an isotropic decay distribution. Reweighting the distributions to be $\sin ^{2} \theta^{*}$ or $1+3 \cos ^{2} \theta^{*}$, as favoured by CLEO data $[8,9]$ changes the relative reconstruction efficiencies by $\pm 9 \%$. This was considered as a systematic error. The almost 
identical kinematics of the different decays make this a good approximation. The efficiencies for the decay chains involving $\mathrm{D}^{0} \rightarrow \mathrm{K}^{-} \pi^{+} \pi^{+} \pi^{-}$and $\mathrm{D}^{0} \rightarrow \mathrm{K}^{-} \rho^{+}$were normalized to the $\mathrm{D}^{0} \rightarrow \mathrm{K}^{-} \pi^{+}$decay mode using the measured ratios of the branching ratios, $2.01 \pm 0.13$ and $2.59 \pm 0.34$ respectively [1]. The errors on these ratios were included as uncertainties on the efficiencies. The overall efficiencies, including the $\mathrm{D}$ and $\mathrm{D}^{*}$ branching ratios, and averaging over modes containing electrons or muons are $0.30 \pm 0.03 \%$ for $\mathrm{D}_{J}^{0} \rightarrow \mathrm{D}^{+} \pi^{-}$ decays, $0.29 \pm 0.02 \%$ for $\mathrm{D}_{J}^{+} \rightarrow \mathrm{D}^{0} \pi^{+}$decays and $0.26 \pm 0.03 \%$ for $\mathrm{D}_{J}^{0} \rightarrow \mathrm{D}^{*+} \pi^{-}$decays.

\subsection{Results}

Using the reconstruction efficiencies and the observed number of signal events in table 5 we determined the $b$ hadron product branching ratios into the different $\mathrm{D}^{(*)} \pi \ell^{-} \mathrm{X}$ final states, listed in table 6 . In this table the states appearing at the higher values of $\Delta_{m}$ in both $\mathrm{D}^{0} \pi^{+}$and $\mathrm{D}^{+} \pi^{-}$have been labelled as the tensor mesons, although these product branching measurements are independent of this assumption. The uncertainties in the reconstruction efficiencies due to Monte Carlo statistics, together with the systematic errors estimated for the inclusive $b \rightarrow \mathrm{D}^{(*)} \ell \mathrm{X}$ production (see table 2 ) and the errors of the $\mathrm{D}^{0}, \mathrm{D}^{+}$and $\mathrm{D}^{*+}$ branching ratios have been included as systematic errors. In addition the effects of detector resolution uncertainties on the $\Delta_{d}$ distribution have been investigated by considering variations on the track $d_{0}$ and $\phi_{0}$ resolutions of $\pm 15 \%$. This contributed a $5 \%$ systematic error. The measured rates for the decays involving a fully reconstructed $\mathrm{D}^{*+} \rightarrow \mathrm{D}^{0} \pi^{+}$or partially reconstructed $\mathrm{D}^{*+} \rightarrow \mathrm{D}^{+} \pi^{0}$ are in agreement and have been averaged.

\begin{tabular}{|c|ll|}
\hline Decay mode & $\begin{array}{l}\text { Branching Ratio } \\
\left(10^{-3}\right)\end{array}$ \\
\hline$B\left(b \rightarrow \mathrm{D}_{J}^{0} \ell^{-} \mathrm{X}\right) \cdot B\left(\mathrm{D}_{J}^{0} \rightarrow \mathrm{D}^{*+} \pi^{-}\right)$ & $\left(\mathrm{D}^{*+} \rightarrow \mathrm{D}^{+} \mathrm{X}\right)$ & $12.4 \pm 3.5_{-4.3}^{+2.2}$ \\
& $\left(\mathrm{D}^{*+} \rightarrow \mathrm{D}^{0} \pi^{+}\right)$ & $5.1 \pm 1.4_{-1.6}^{+1.2}$ \\
& Average & $6.1 \pm 1.3 \pm 1.3$ \\
\hline$B\left(b \rightarrow \mathrm{D}_{2}^{* 0}(2460) \ell^{-} \mathrm{X}\right) \cdot B\left(\mathrm{D}_{2}^{* 0}(2460) \rightarrow \mathrm{D}^{+} \pi^{-}\right)$ & & $1.6 \pm 0.7 \pm 0.3$ \\
\hline$B\left(b \rightarrow \mathrm{D}_{J}^{+} \ell^{-} \mathrm{X}\right) \cdot B\left(\mathrm{D}_{J}^{+} \rightarrow \mathrm{D}^{0} \pi^{+}\right)$ & & $7.0 \pm 1.9_{-1.3}^{+1.2}$ \\
\hline$B\left(b \rightarrow \mathrm{D}_{2}^{*+}(2460) \ell^{-} \mathrm{X}\right) \cdot B\left(\mathrm{D}_{2}^{*+}(2460) \rightarrow \mathrm{D}^{0} \pi^{+}\right)$ & & $4.2 \pm 1.3_{-1.2}^{+0.7}$ \\
\hline
\end{tabular}

Table 6: Product branching ratio measurements. The states indicated by $\mathrm{D}_{J}$ represent an unresolved mixture of the pseudovector and tensor states.

If one assumes that the observed $\mathrm{D}_{J}$ signals are due solely to the presence of the $\mathrm{D}_{1}^{0}(2420), \mathrm{D}_{1}^{+}(2420), \mathrm{D}_{2}^{* 0}(2460)$ and $\mathrm{D}_{2}^{*+}(2460)$ mesons then one can estimate the $b$ hadron semileptonic branching ratio into these states. In order to do this the $\mathrm{D}_{J}$ branching ratios given in table 3 were assumed. The production rates of the tensor states were derived directly from the data using the $46 \%$ branching ratio to the observed peaks. The pseudovector production rates were obtained by subtracting the estimated contributions 


\begin{tabular}{|c|c|}
\hline Decay mode & $\begin{array}{c}\text { Branching Ratio } \\
\left(10^{-3}\right)\end{array}$ \\
\hline$B\left(b \rightarrow \mathrm{D}_{1}^{0}(2420) \ell^{-} \mathrm{X}\right)$ & $8.1 \pm 2.0 \pm 1.9$ \\
\hline$B\left(b \rightarrow \mathrm{D}_{2}^{* 0}(2460) \ell^{-} \mathrm{X}\right)$ & $3.5 \pm 1.4 \pm 0.7$ \\
\hline$B\left(b \rightarrow \mathrm{D}_{1}^{+}(2420) \ell^{-} \mathrm{X}\right)$ & $7.8 \pm 2.8 \pm 1.8$ \\
\hline$B\left(b \rightarrow \mathrm{D}_{2}^{*+}(2460) \ell^{-} \mathrm{X}\right)$ & $9.0 \pm 2.7 \pm 2.1$ \\
\hline
\end{tabular}

Table 7: Estimated $b$ hadron semileptonic branching ratios to the four narrow $\mathrm{D}_{J}$ states.

of the tensor mesons to the peaks at $\Delta_{m} \sim 440 \mathrm{MeV}$ and assuming the remaining signals were due to the pseudovector states with a $67 \%$ branching ratio. The resulting branching ratios are listed in table 7 . No additional errors have been added for the uncertainties involved in deriving these results from the measurements listed in table 6 . It should be noted that some models [25] predict significant decay fractions of the $\mathrm{D}_{J}$ mesons into $\mathrm{D}^{(*)} \rho$ states. This would increase the corresponding production rates.

Our measurements indicate that the decays to the narrow P-wave states form a significant fraction of the total semileptonic decay width, although similar rates for the decays involving the wide $\mathrm{P}$-wave mesons, higher mass states or non-resonant $\mathrm{D}^{*} \pi$ combinations are not excluded.

Comparing the estimated rates to the narrow $\mathrm{P}$-wave states given in table 7 with our measurement of the inclusive rate measurements given in section 5.4 implies that these decays account for $34 \pm 7 \%$ of the total.

\section{Conclusions}

From a sample of approximately 1300 semileptonic B mesons decaying to charm mesons we determined the product branching ratios:

$$
\begin{aligned}
B\left(b \rightarrow \mathrm{D}^{+} \ell X\right) \cdot B\left(\mathrm{D}^{+} \rightarrow \mathrm{K}^{-} \pi^{+} \pi^{+}\right) & =(1.82 \pm 0.20 \pm 0.12) \times 10^{-3}, \\
B\left(b \rightarrow \mathrm{D}^{0} \ell X\right) \cdot B\left(\mathrm{D}^{0} \rightarrow \mathrm{K}^{-} \pi^{+}\right) & =(2.52 \pm 0.14 \pm 0.17) \times 10^{-3}, \\
B\left(b \rightarrow \mathrm{D}^{*+} \ell X\right) \cdot B\left(\mathrm{D}^{*+} \rightarrow \mathrm{D}^{0} \pi^{+}\right) \cdot B\left(\mathrm{D}^{0} \rightarrow \mathrm{K}^{-} \pi^{+}\right) & =(7.53 \pm 0.47 \pm 0.56) \times 10^{-4} .
\end{aligned}
$$

These measurements are in agreement with results obtained previously by the DELPHI collaboration [26], but represent a significant improvement in precision.

Combining the first two results with measurements from CLEO we determined the fraction of $\mathrm{b}$ quarks that fragment to either $\mathrm{a}^{0}$ or $\mathrm{B}^{+}$meson to be:

$$
f\left(b \rightarrow \mathrm{B}^{0}\right)+f\left(b \rightarrow \mathrm{B}^{+}\right)=0.81 \pm 0.07 \pm 0.09 .
$$

The observation of $44 \pm 8_{-7}^{+3} \mathrm{D}_{J}^{0}$ and $48 \pm 10_{-6}^{+3} \mathrm{D}_{J}^{+}$candidates provides evidence for the production of both charged and neutral $\mathrm{P}$-wave charmed mesons in semileptonic $\mathrm{B}$ decays. 
Interpreting these signals as decays of the narrow P-wave states we measure the following product branching ratios:

$$
\begin{aligned}
B\left(b \rightarrow \mathrm{D}_{J}^{0} \ell^{-} \mathrm{X}\right) \cdot B\left(\mathrm{D}_{J}^{0} \rightarrow \mathrm{D}^{*+} \pi^{-}\right) & =(6.1 \pm 1.3 \pm 1.3) \times 10^{-3} \\
B\left(b \rightarrow \mathrm{D}_{2}^{* 0}(2460) \ell^{-} \mathrm{X}\right) \cdot B\left(\mathrm{D}_{2}^{* 0}(2460) \rightarrow \mathrm{D}^{+} \pi^{-}\right) & =(1.6 \pm 0.7 \pm 0.3) \times 10^{-3} \\
B\left(b \rightarrow \mathrm{D}_{J}^{+} \ell^{-} \mathrm{X}\right) \cdot B\left(\mathrm{D}_{J}^{+} \rightarrow \mathrm{D}^{0} \pi^{+}\right) & =\left(7.0 \pm 1.9_{-1.3}^{+1.2}\right) \times 10^{-3} \\
B\left(b \rightarrow \mathrm{D}_{2}^{*+}(2460) \ell^{-} \mathrm{X}\right) \cdot B\left(\mathrm{D}_{2}^{*+}(2460) \rightarrow \mathrm{D}^{0} \pi^{+}\right) & =\left(4.2 \pm 1.3_{-1.2}^{+0.7}\right) \times 10^{-3}
\end{aligned}
$$

This is the first evidence for semileptonic B decays to charged P-wave charm states, and the first measurement separating the pseudovector and tensor meson components. The measured production rates of the neutral states are consistent with the ARGUS [10] and the recent ALEPH [27] results. Comparison with our measurements of the inclusive $b \rightarrow \mathrm{D}^{0} \ell \mathrm{X}$ and $b \rightarrow \mathrm{D}^{+} \ell \mathrm{X}$ rates indicates that together these modes form $34 \pm 7 \%$ of charmed semileptonic $\mathrm{B}^{0}$ and $\mathrm{B}^{+}$decays. The branching ratios to these decay modes are thus somewhat larger than the predictions of several theoretical models [2,3].

\section{Acknowledgements:}

It is a pleasure to thank the SL Division for the efficient operation of the LEP accelerator, the precise information on the absolute energy, and their continuing close cooperation with our experimental group. In addition to the support staff at our own institutions we are pleased to acknowledge the

Department of Energy, USA,

National Science Foundation, USA,

Particle Physics and Astronomy Research Council, UK,

Natural Sciences and Engineering Research Council, Canada,

Fussefeld Foundation,

Israel Ministry of Science,

Israel Science Foundation, administered by the Israel Academy of Science and Humanities.

Minerva Gesellschaft,

Japanese Ministry of Education, Science and Culture (the Monbusho) and a grant under the Monbusho International Science Research Program,

German Israeli Bi-national Science Foundation (GIF),

Direction des Sciences de la Matière du Commissariat à l'Energie Atomique, France,

Bundesministerium für Forschung und Technologie, Germany,

National Research Council of Canada,

A.P. Sloan Foundation and Junta Nacional de Investigação Científica e Tecnológica, Portugal. 


\section{References}

[1] Particle Data Group, L. Montanet et al., Phys. Rev. D50 (1994).

[2] N. Isgur, D. Scora, B. Grinstein and M. Wise, Phys. Rev. D39 (1989) 799.

[3] P. Colangelo, G. Nardulli and N. Paver, Phys. Lett. B293 (1992) 207.

[4] I. Bigi et al., Phys. Lett. B323 (1994) 408.

[5] ARGUS Collaboration, H. Albrecht et al., Phys. Lett. B221 (1989) 422;

ARGUS Collaboration, H. Albrecht et al., Phys. Lett. B232 (1989) 398;

CLEO Collaboration, P. Avery et al., Phys. Rev. D41 (1990) 774.

[6] E691 Collaboration, J. C. Anjos et al., Phys. Rev. Lett. 62 (1989) 1717.

[7] E687 Collaboration, P. L. Frabetti et al., Phys. Rev. Lett. 72 (1994) 324;

ARGUS Collaboration, H. Albrecht et al., Phys. Lett. B231 (1989) 208.

[8] CLEO Collaboration, P. Avery et al., Phys. Lett. B331 (1994) 236.

[9] CLEO Collaboration, T. Bergfeld et al., Phys. Lett. B340 (1994) 194.

[10] ARGUS Collaboration, H. Albrecht et al., Z. Phys. C57 (1993) 533.

[11] OPAL Collaboration, K. Ahmet et al., Nucl. Instrum. Methods A305 (1991) 275.

[12] P. P. Allport et al., Nucl. Instrum. Methods A324 (1993) 34.

[13] P. P. Allport et al., Nucl. Instrum. Methods A346 (1994) 476.

[14] M. Hauschild et al., Nucl. Instrum. Methods A314 (1992) 74.

[15] OPAL Collaboration, R. Akers et al., Z. Phys. C60 (1993) 199.

[16] OPAL Collaboration, P. D. Acton et al., Z. Phys. C58 (1993) 523.

[17] J. Allison et al., Nucl. Instrum. Methods A317 (1992) 47.

[18] T. Sjöstrand, Comp. Phys. Comm. 39 (1986) 347;

M. Bengtsson and T. Sjöstrand, Comp. Phys. Comm. 43 (1987) 367;

M. Bengtsson and T. Sjöstrand, Nucl. Phys. B 289 (1987) 810.

[19] OPAL Collaboration, P. D. Acton et al., Phys. Lett. B307 (1993) 247.

[20] C.Peterson, D.Schlatter, I.Schmitt and P.M.Zerwas, Phys. Rev. D 27 (1983) 105.

[21] CLEO Collaboration, R. Fulton et al., Phys. Rev. D43 (1991) 651.

[22] OPAL Collaboration, G. Alexander et al., Z. Phys. C52 (1991) 175.

[23] OPAL Collaboration, R. Akers et al., CERN-PPE/94-106.

[24] OPAL Collaboration, P. D. Acton et al., Phys. Lett. B295 (1992) 357. 
[25] J. L. Rosner, Comm. Nucl. Part. Phys. (1986) 109;

S. Godfrey and R. Kokoski, Phys. Rev. D43 (1991) 1679;

E. J. Eichten, C. T. Hill and C. Quigg, Phys. Rev. Lett. 71 (1993) 4116.

[26] DELPHI Collaboration, P. Abreu et al., Z. Phys. C57 (1993) 181.

[27] ALEPH Collaboration, D. Buskulic et al., CERN-PPE/94-173 (1994). 


\section{Figure Captions}

1. $\mathrm{D}^{0}$ mass distributions for events containing a $\ell^{-}$and a) $\mathrm{K}^{-} \pi^{+}$, b) $\mathrm{K}^{-} \pi^{+} \pi^{+} \pi^{-}$. The fits shown are the sum of Gaussians and a second order polynomial function.

2. $\mathrm{D}^{+}$mass distribution for events containing a $\ell^{-}$and $\mathrm{K}^{-} \pi^{+} \pi^{+}$. The fit shown is the sum of two Gaussians and a second order polynomial function.

3. $\mathrm{D}^{0}$ mass distribution for $\mathrm{D}^{*+} \rightarrow \mathrm{D}^{0} \pi^{+}$events containing a $\ell^{-}$and a) $\mathrm{K}^{-} \pi^{+}$, b) $\mathrm{K}^{-} \pi^{+} \pi^{+} \pi^{-}$. The fits shown are the sum of Gaussians and a second order polynomial function.

4. Monte Carlo and data comparison of $m_{\mathrm{D}^{(*)} \ell}$. a) $b \rightarrow \mathrm{D}^{0} \ell X$ events, b) $b \rightarrow \mathrm{D}^{+} \ell X$ events and c) $b \rightarrow \mathrm{D}^{*+} \ell X$ events. The histograms are the generator level Monte Carlo distributions and the points are efficiency corrected data after background subtraction. The dotted line on each plot indicates the experimental lower limit due to the selection criteria applied.

5. Monte Carlo and data comparison of $E_{\mathrm{D}^{(*)} \ell}$. a) $b \rightarrow \mathrm{D}^{0} \ell X$ events, b) $b \rightarrow \mathrm{D}^{+} \ell X$ events and c) $b \rightarrow \mathrm{D}^{*+} \ell X$ events. The histograms are the generator level Monte Carlo distributions and the points are efficiency corrected data after background subtraction. The dotted line on each plot indicates the experimental lower limit due to the selection criteria applied.

6. Distribution of $\Delta_{m}$ for the Monte Carlo sample of $b \rightarrow \mathrm{D}^{0} \ell^{-} \mathrm{X}$ decays a) before $\mathrm{D}_{J}^{+}$selection and $\mathrm{b}$ ) after all selection criteria have been applied. For both plots the points with errors are the total number of entries and the curves are fits to the data. In a) the shaded histogram shows the signal events whilst in b) the shaded histogram corresponds to the residual background.

7. $\Delta_{m}$ distributions satisfying the $\mathrm{D}_{J}$ selection criteria for a) $\mathrm{D}^{*+} \pi^{-}$combinations, $\mathrm{b}$ ) $\mathrm{D}^{+} \pi^{-}$combinations, c) $\mathrm{D}^{0} \pi^{+}$combinations.

8. $\Delta_{m}$ distributions with $\Delta_{d}<-0.5$ and with no requirements on $\left|d_{v x} / \sigma_{v x}\right|$ for a) $\mathrm{D}^{*+} \pi^{-}$combinations, b) $\mathrm{D}^{+} \pi^{-}$combinations, c) $\mathrm{D}^{0} \pi^{+}$combinations.

9. $\Delta_{m}$ distributions satisfying the $\mathrm{D}_{J}$ selection criteria for a) $\mathrm{D}^{*+} \pi^{+}$combinations, $\mathrm{b}$ ) $\mathrm{D}^{+} \pi^{+}$combinations, c) $\mathrm{D}^{0} \pi^{-}$combinations. 

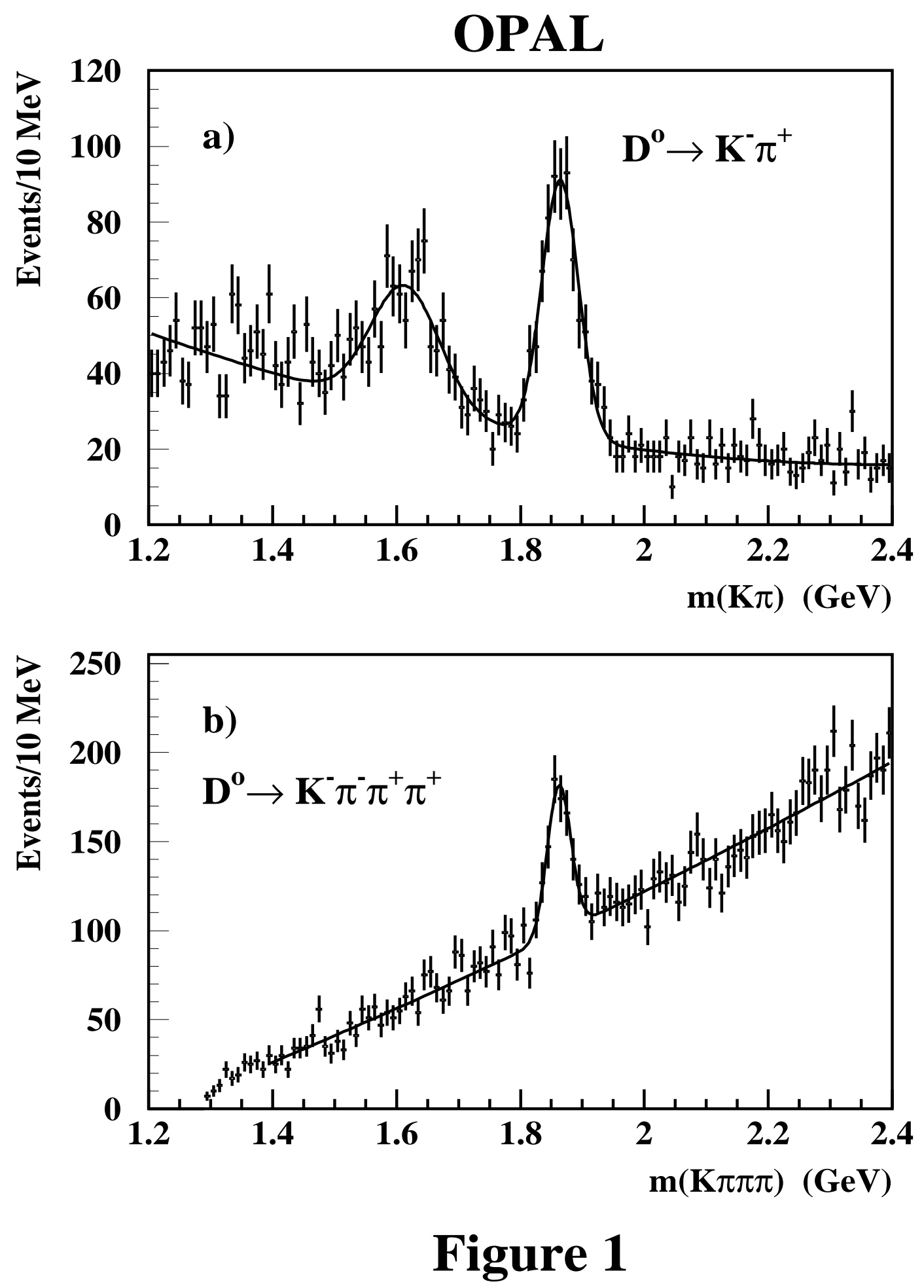


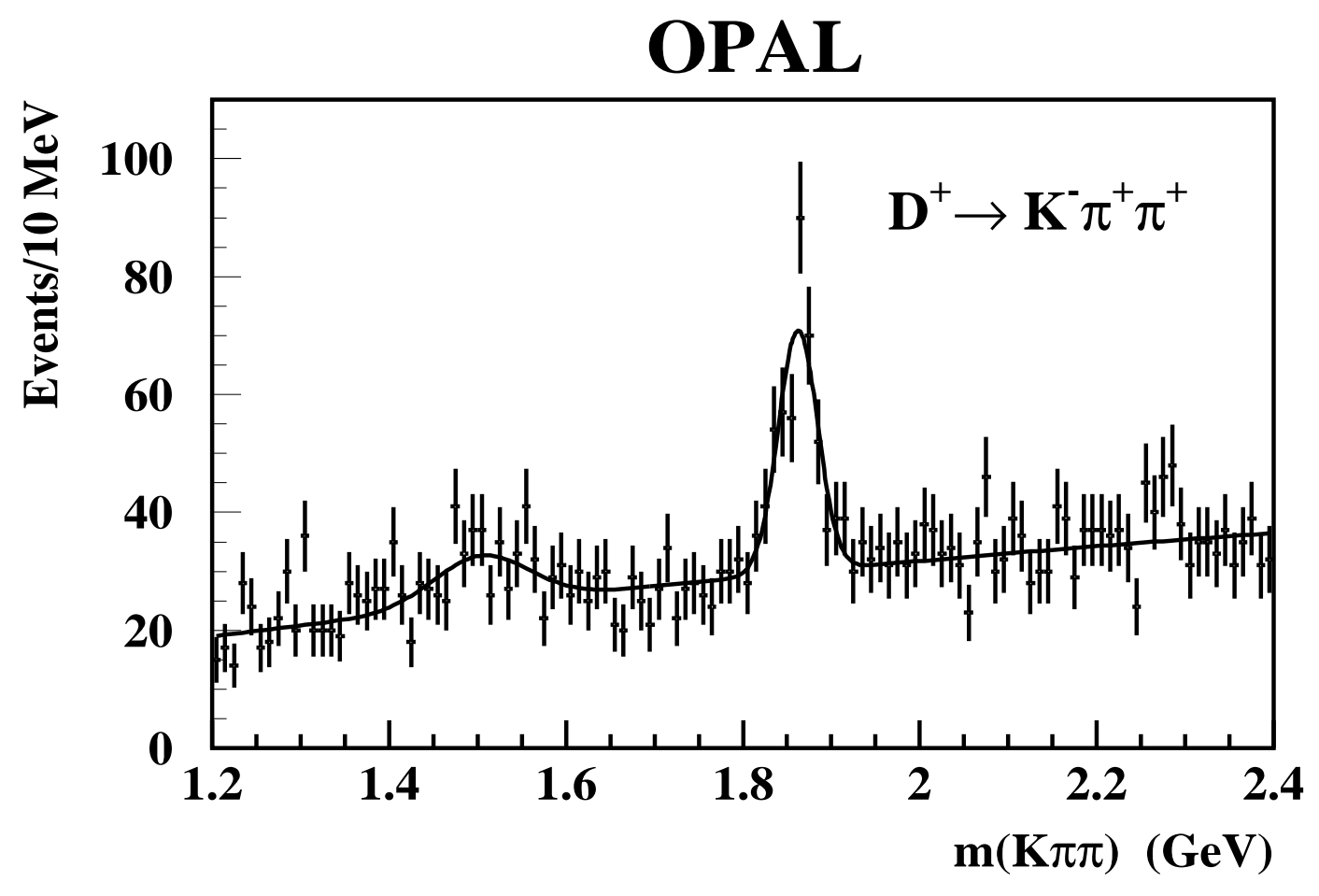

Figure 2 

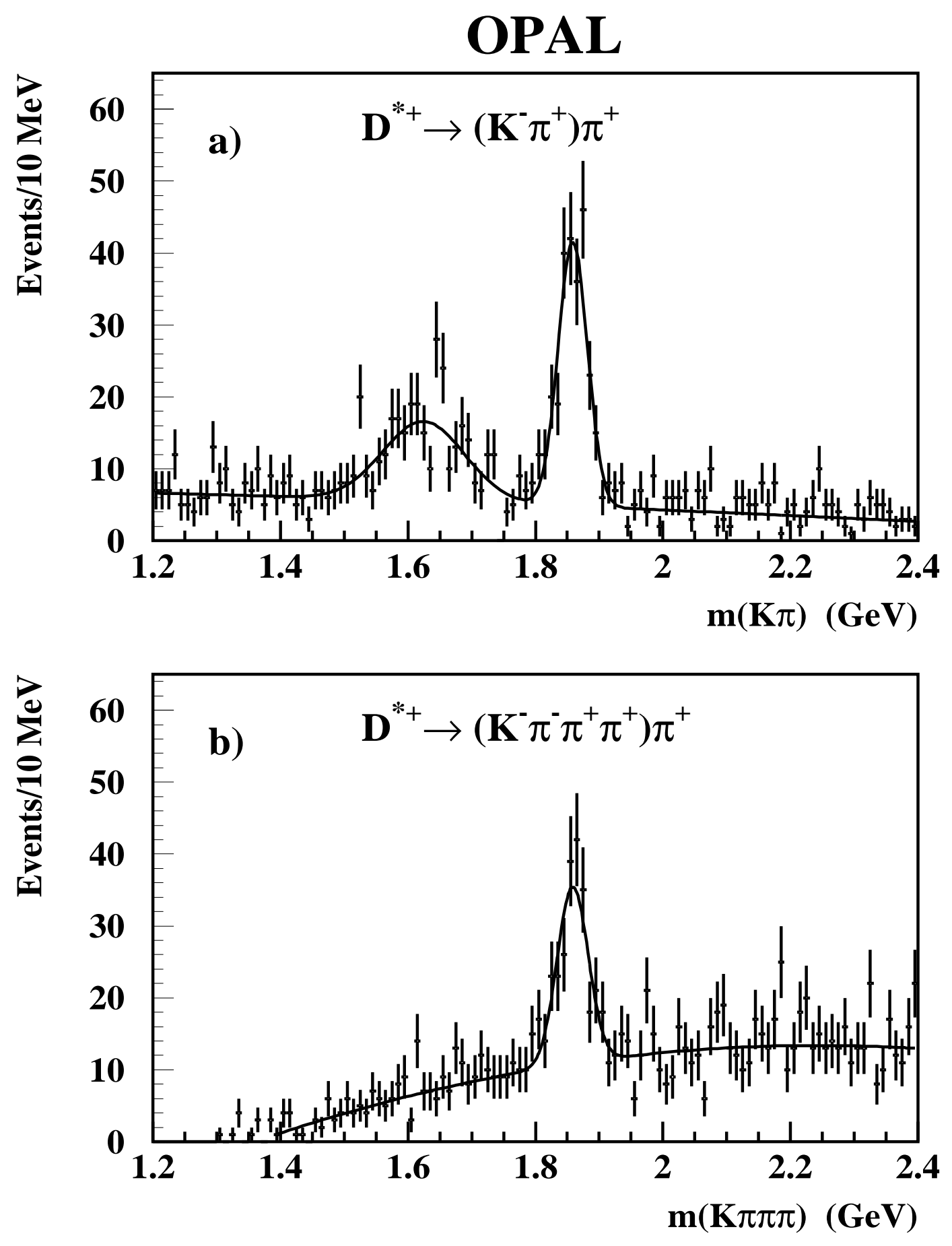

Figure 3 
OPAL
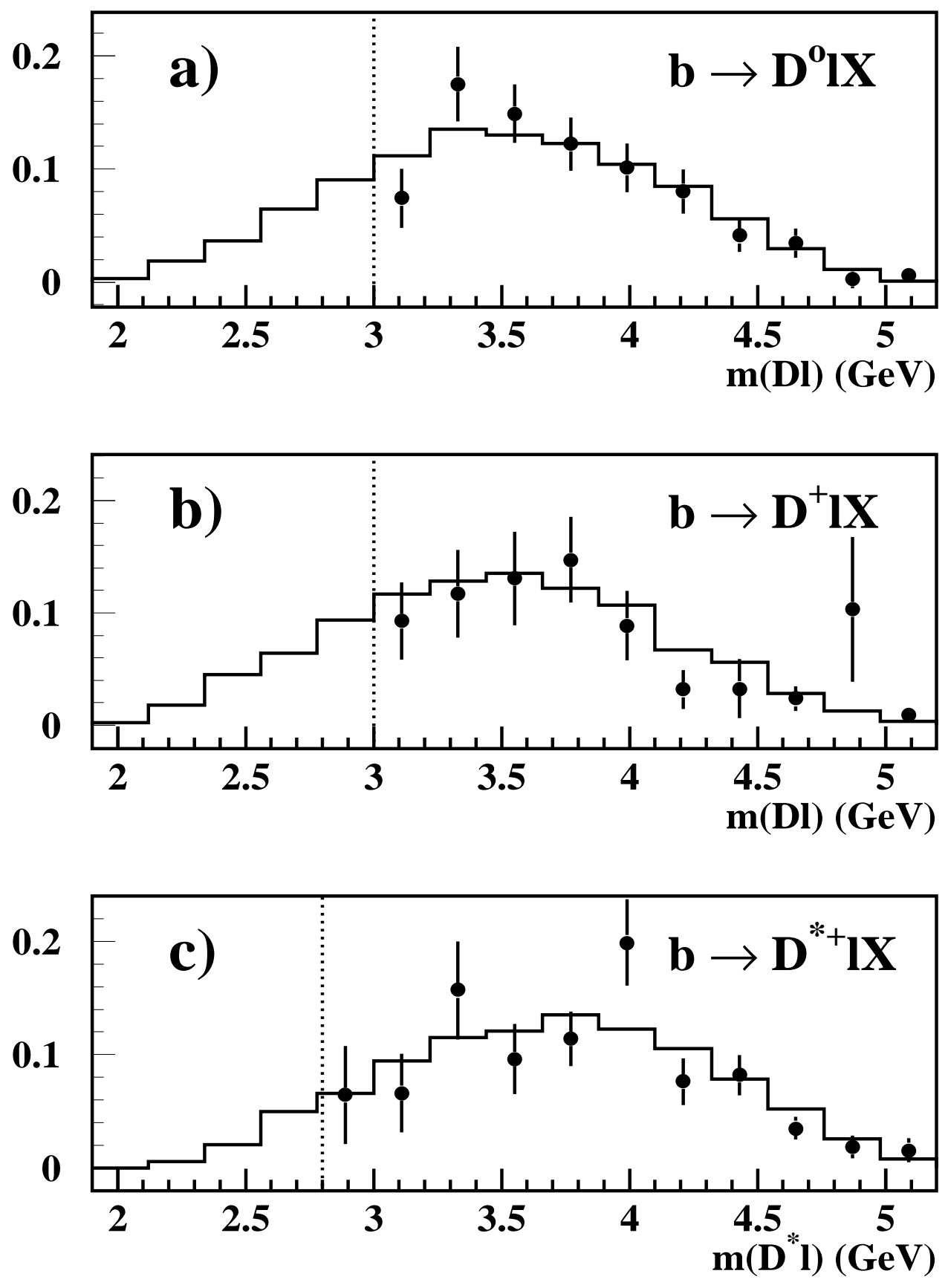

Figure 4 
OPAL
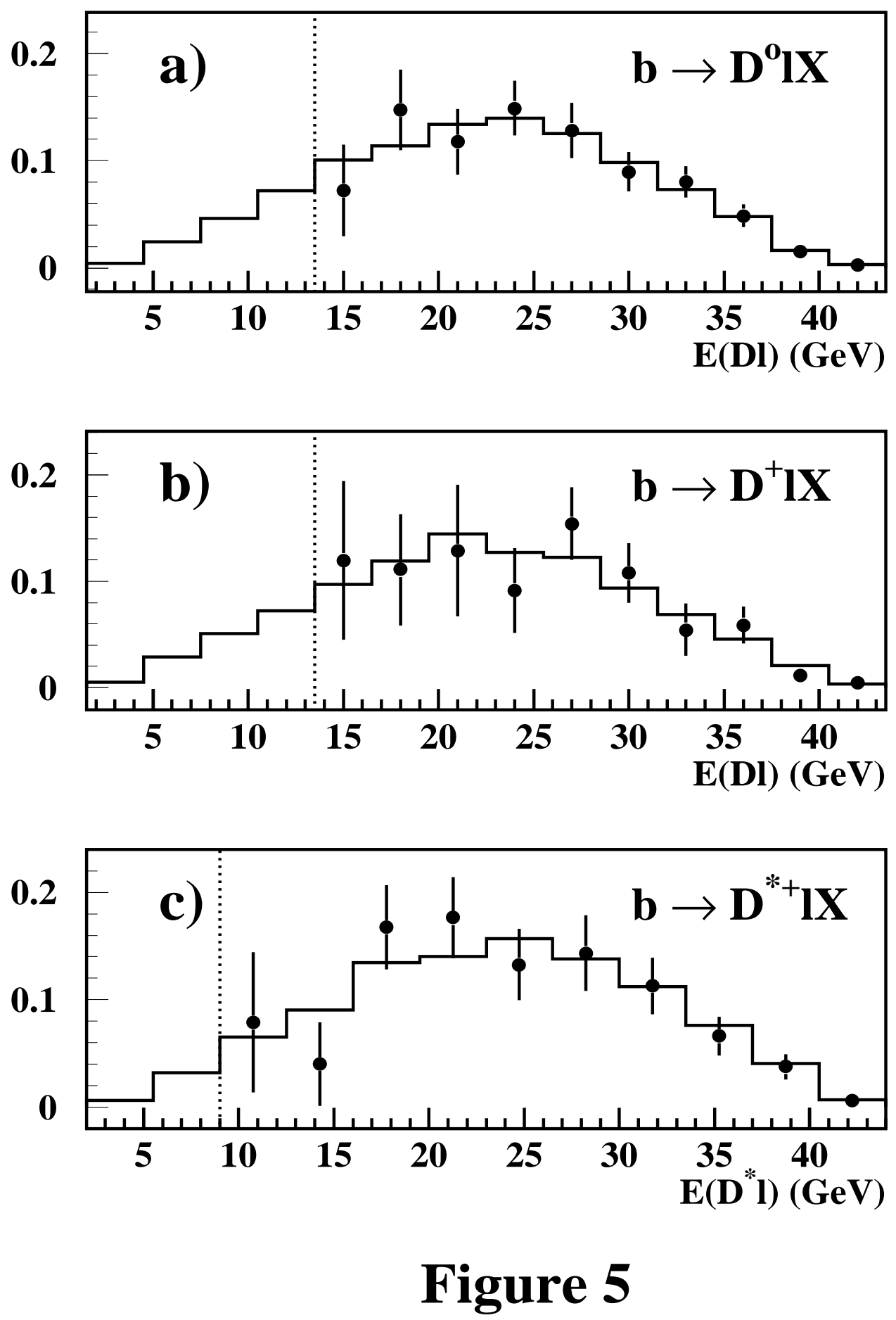

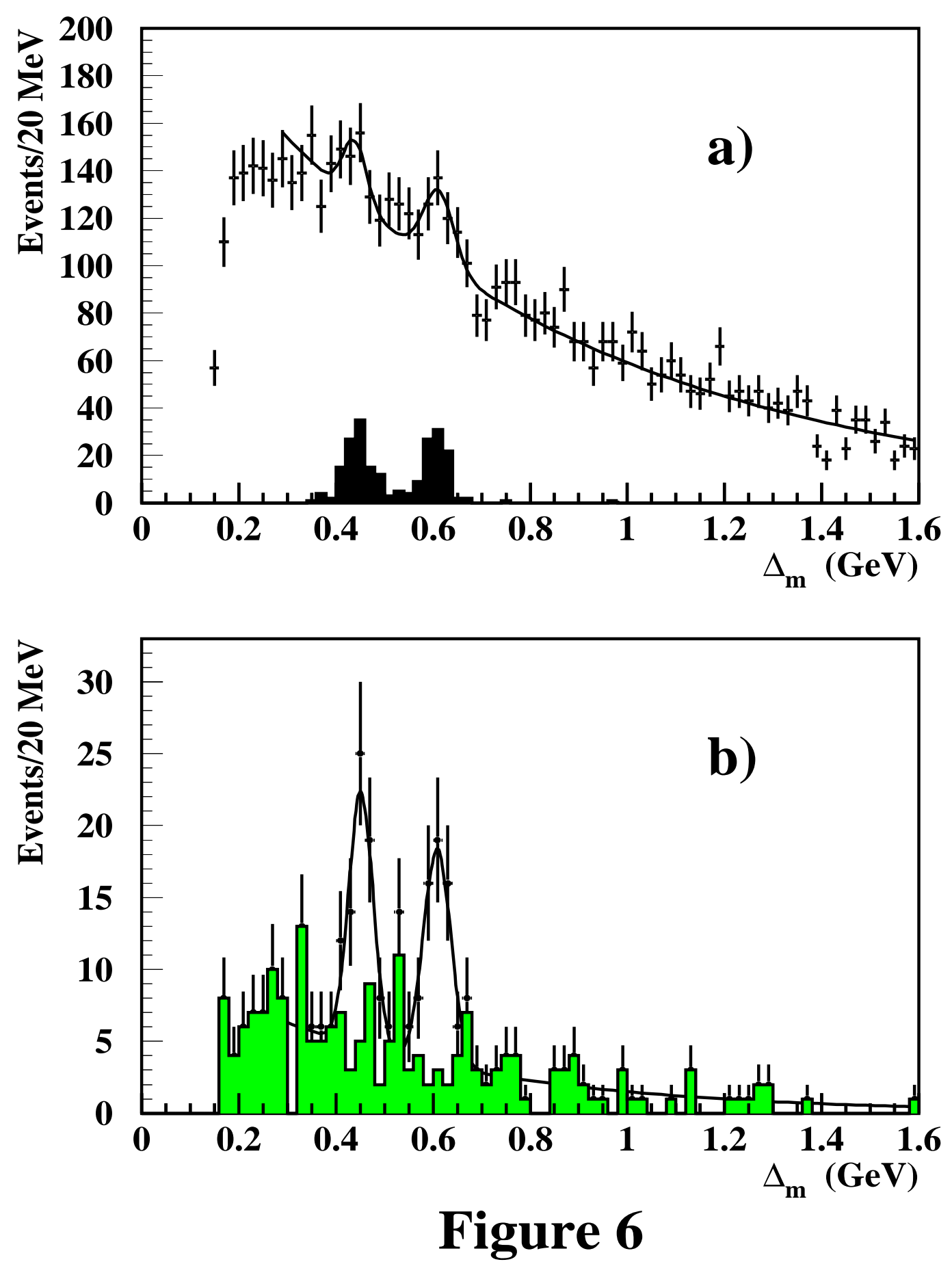
OPAL
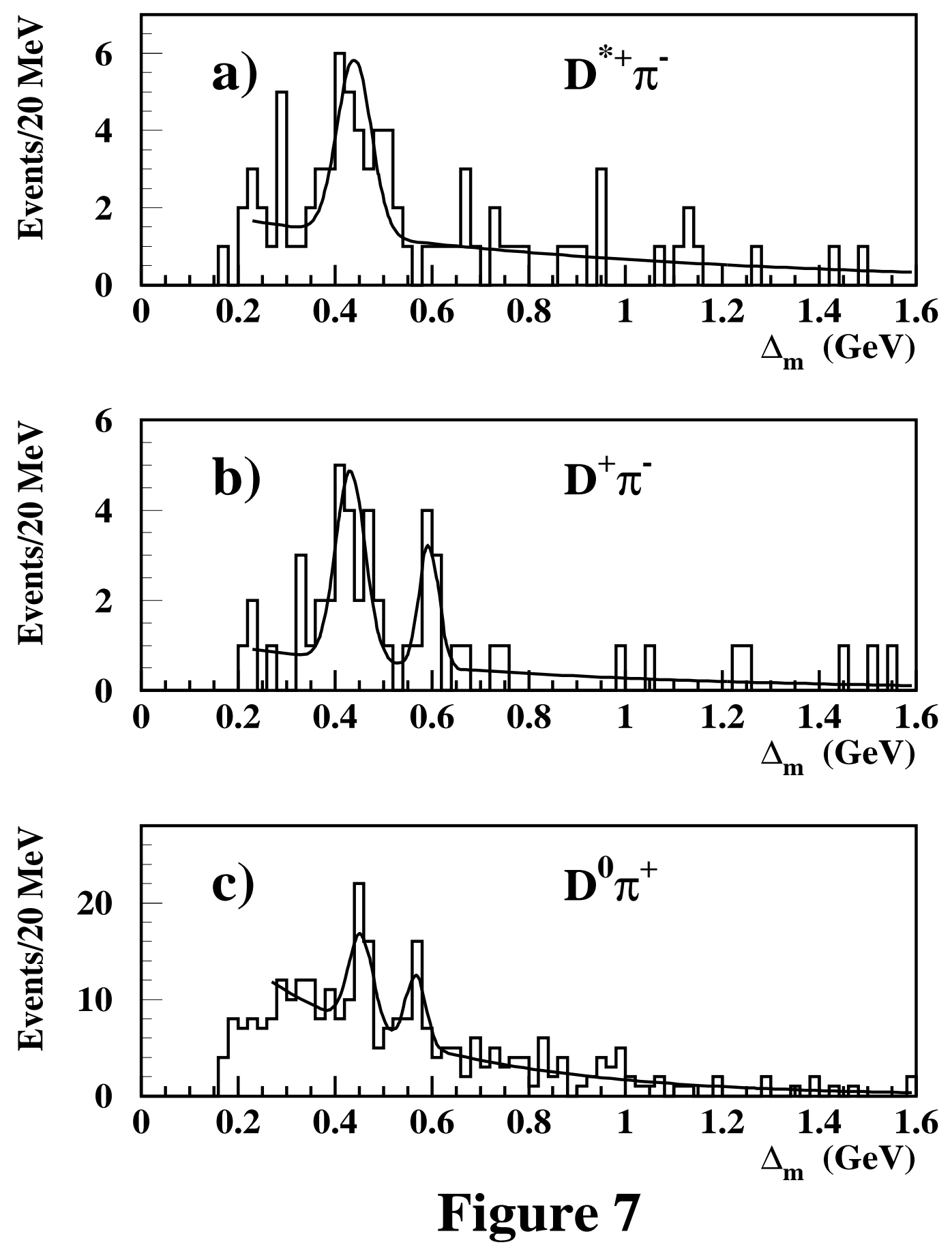
OPAL
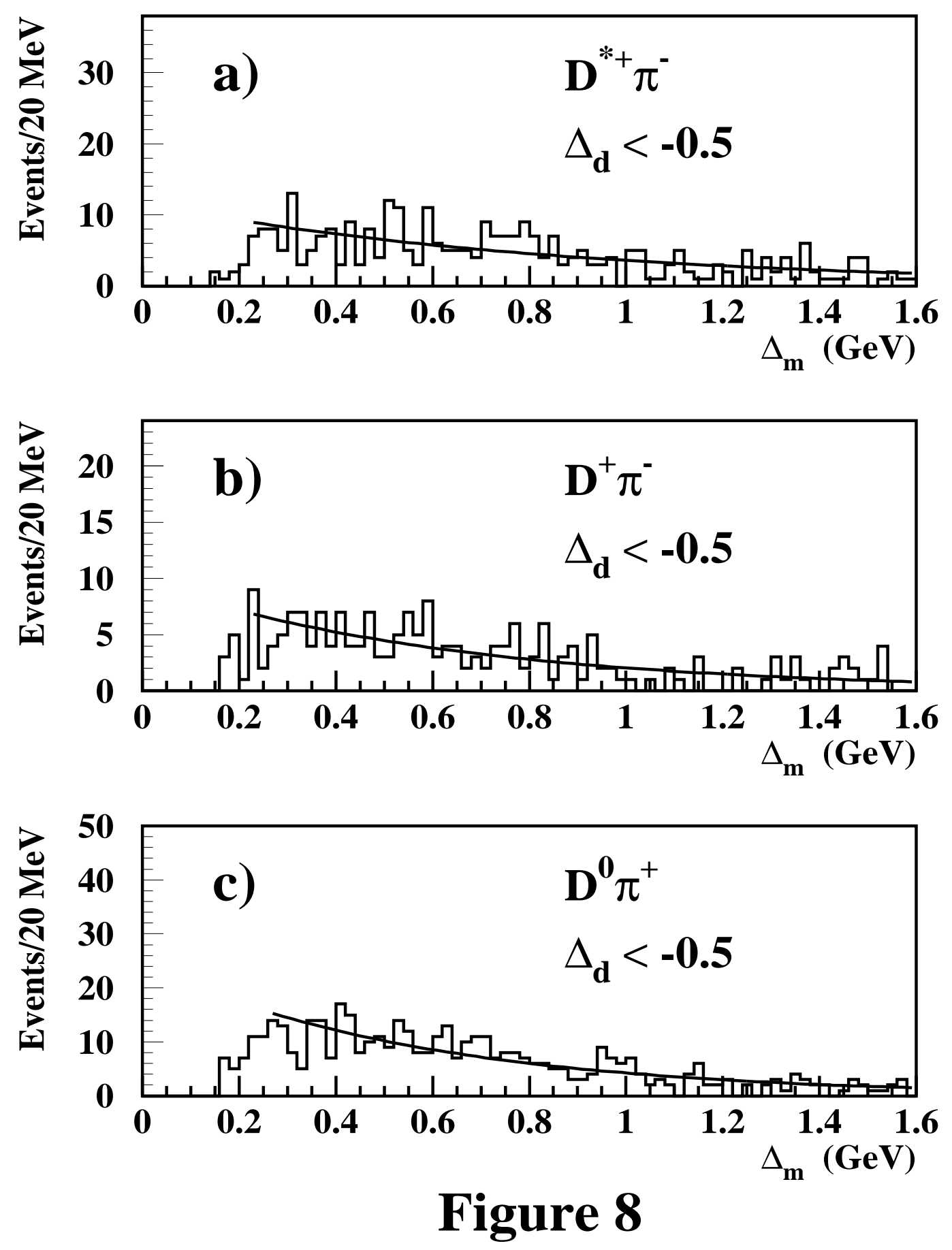
OPAL
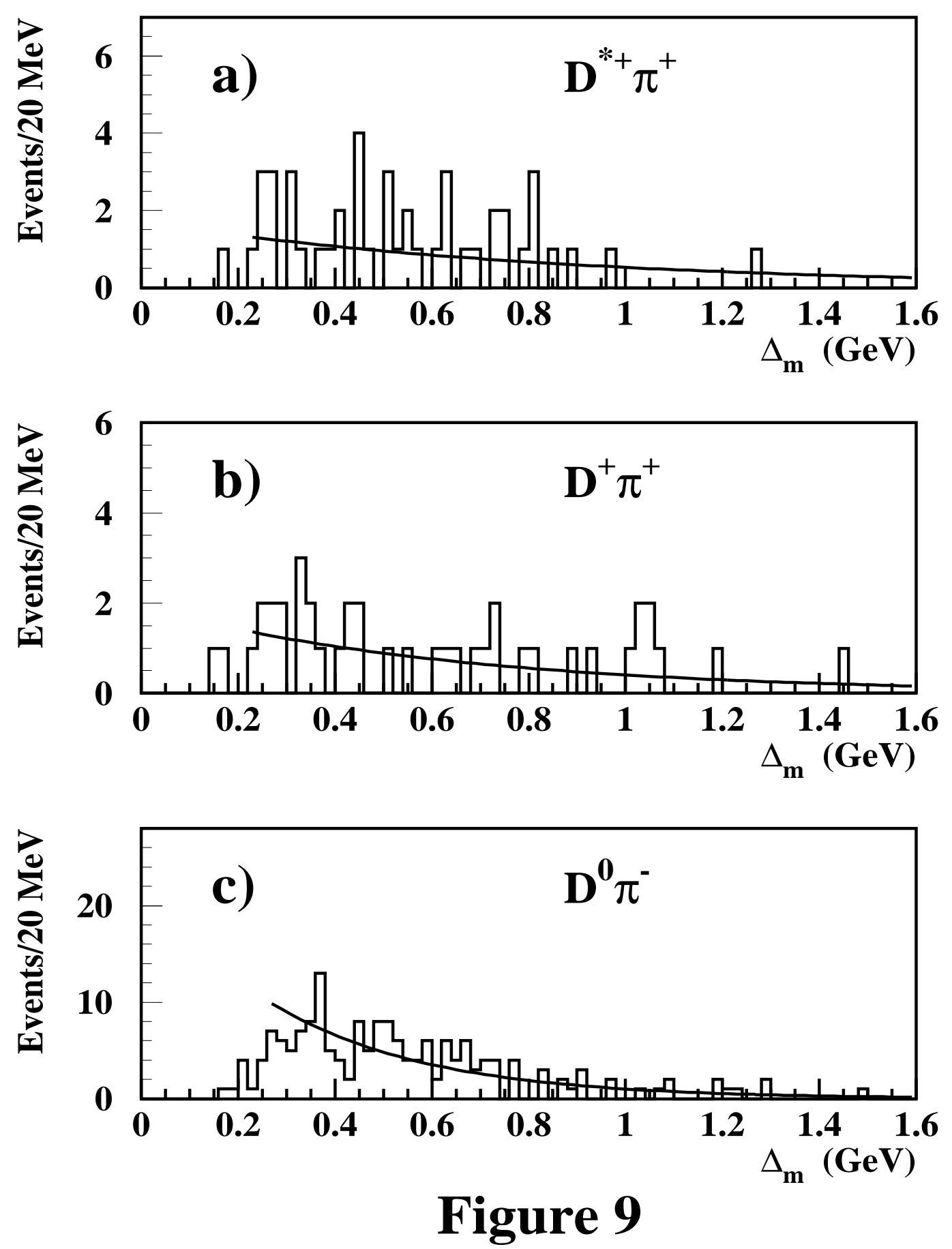